\title{
NO mediates mural cell recruitment and vessel morphogenesis in murine melanomas and tissue-engineered blood vessels
}

\author{
Satoshi Kashiwagi, ${ }^{1}$ Yotaro Izumi, ${ }^{1}$ Takeshi Gohongi, ${ }^{1}$ Zoe N. Demou, ${ }^{1}$ Lei Xu, ${ }^{1}$ Paul L. Huang, ${ }^{2}$ \\ Donald G. Buerk, ${ }^{3}$ Lance L. Munn, ${ }^{1}$ Rakesh K. Jain, ${ }^{1}$ and Dai Fukumura ${ }^{1}$

\begin{abstract}
${ }^{1}$ Edwin L. Steele Laboratory, Department of Radiation Oncology, and ${ }^{2}$ Cardiovascular Research Center, Department of Medicine, Massachusetts General Hospital and Harvard Medical School, Boston, Massachusetts, USA. ${ }^{3}$ Departments of Physiology and Bioengineering,
\end{abstract} \\ University of Pennsylvania School of Medicine, Philadelphia, Pennsylvania, USA.
}

\begin{abstract}
NO has been shown to mediate angiogenesis; however, its role in vessel morphogenesis and maturation is not known. Using intravital microscopy, histological analysis, $\alpha$-smooth muscle actin and chondroitin sulfate proteoglycan 4 staining, microsensor NO measurements, and an NO synthase (NOS) inhibitor, we found that NO mediates mural cell coverage as well as vessel branching and longitudinal extension but not the circumferential growth of blood vessels in B16 murine melanomas. NO-sensitive fluorescent probe 4,5-diaminofluorescein imaging, NOS immunostaining, and the use of NOS-deficient mice revealed that eNOS in vascular endothelial cells is the predominant source of NO and induces these effects. To further dissect the role of NO in mural cell recruitment and vascular morphogenesis, we performed a series of independent analyses. Transwell and under-agarose migration assays demonstrated that endothelial cell-derived NO induces directional migration of mural cell precursors toward endothelial cells. An in vivo tissue-engineered blood vessel model revealed that NO mediates endothelial-mural cell interaction prior to vessel perfusion and also induces recruitment of mural cells to angiogenic vessels, vessel branching, and longitudinal extension and subsequent stabilization of the vessels. These data indicate that endothelial cell-derived NO induces mural cell recruitment as well as subsequent morphogenesis and stabilization of angiogenic vessels.
\end{abstract}

\section{Introduction}

$\mathrm{NO}$ is a multifunctional gaseous molecule that regulates various physiological functions, including blood flow, vascular permeability, and leukocyte-endothelial interaction (1-4). Furthermore, NO has been shown to promote angiogenesis (the development of new blood vessels derived from existing vessels) and vasculogenesis (de novo blood vessel formation from progenitor cells), indispensable processes for tissue growth $(4,5)$. NO is not only recognized as proangiogenic by itself; it also mediates the functions of many angiogenic factors $(4,5)$. For example, VEGF $(6)$, angiopoietin-1 (7), sphingosine-1-phosphate (S1P) (8), and shear stress (9) are known to activate endothelial NO production through the $\mathrm{PI} 3 \mathrm{~K} / \mathrm{Akt}$ pathway and to induce endothelial cell proliferation, migration, survival, and tube formation. $\mathrm{NO}$ is also an important modulator for the expression of endogenous angiogenic factors such as VEGF and basic FGF $(10,11)$. Involvement of NO in tumor angiogenesis has been documented (12-14). However, the site of $\mathrm{NO}$ production and the relationship between actual tissue NO

Nonstandard abbreviations used: 4-AF, 4-aminofluorescein; BME, basal medium Eagle; cGMP, cyclic GMP; DAF-2, 4,5-diaminofluorescein; DAF-2T, DAF-2 triazole; D-NMMA, $\mathrm{N}^{\mathrm{G}}$-monomethyl-D-arginine monoacetate; DsRed, red fluorescent protein from Discosoma; EDG-1, endothelial differentiation sphingolipid G-protein-coupled receptor 1; eGFP, enhanced GFP; EGM, endothelial growth media; HUVEC, human umbilical vein endothelial cell; $\mathrm{L}-\mathrm{NMMA}, \mathrm{N}^{\mathrm{G}}$-monomethyl-L-arginine monoacetate; MECA, mouse endothelial cell-specific differentiation antigen; MPLSM, multiphoton laser-scanning microscopy; NG2, chondroitin sulfate proteoglycan 4; nNOS, neuronal NOS; NOS, NO synthase; PCNA, proliferating cell nuclear antigen.

Conflict of interest: The authors have declared that no conflict of interest exists.

Citation for this article: J. Clin. Invest. 115:1816-1827 (2005).

doi:10.1172/JCI24015. level and angiogenic activity, the resulting vessel architecture, and vascular function in tumors are not known.

There are 3 isoforms of NO synthase (NOS): neuronal NOS (nNOS), also referred to as type I NOS; iNOS, also referred to as type II NOS; and eNOS, also referred to as type III NOS. These 3 isoforms of NOS are distributed and regulated differently (2). Following studies of NOS-deficient mice, it has been reported that eNOS mediates collateral vessel formation in ischemic limb and capillary ingrowth in both Matrigel implants and transplanted murine tumors $(15,16)$. Furthermore, of the 3 isoforms of NOS, it is eNOS that predominantly mediates VEGF-induced angiogenesis and retinal neovascularization during oxygen-induced ischemic retinopathy $(17,18)$. On the other hand, iNOS and nNOS mediate choroidal neovascularization in a laser-induced Bruch's membrane rupture model, while eNOS does not do so (18). In tumors, both eNOS and iNOS have been shown to mediate angiogenesis $(12,19,20)$. Different isoforms of NOS may be involved in angiogenesis depending on the context. However, the relative contributions and distinct role of each NOS in any given tumor model have not been documented.

It is well recognized that blood vessels consist of 2 distinct types of cells, endothelial cells and mural cells, and that recruitment of mural cells, such as pericytes and vascular SMCs, is an important step in angiogenesis, vascular morphogenesis, and vessel maturation (21-23). However, the role of $\mathrm{NO}$ in this process has been unexplored. The incidence and mortality rate of melanomas are increasing annually by $2-3 \%(24)$. Since the interaction between host and tumor is an important determinant of melanoma development and progression (25), we used the dorsal skin chamber and cranial window models, which provide unique opportunities to study melanoma in its orthotopic primary and metastatic environments, respectively $(26,27)$. 
Table 1

Tissue NO level, angiogenesis, and vessel morphology of B16 melanomas

\begin{tabular}{lllcc}
\hline & & & & \\
& \multicolumn{2}{c}{ Dorsal skin chamber } & \multicolumn{2}{c}{ Cranial window } \\
& B16 F1 & B16 F10 & B16 F1 & B16 F10 \\
Tissue N0 level $(\mu \mathrm{M})$ & $1.5 \pm 0.2$ & $2.4 \pm 0.3^{\mathrm{A}}$ & $2.5 \pm 0.1$ & $3.1 \pm 0.1^{\mathrm{A}}$ \\
Vessel density $\left(\mathrm{cm} / \mathrm{cm}^{2}\right)$ & $42 \pm 5$ & $84 \pm 10^{\mathrm{A}}$ & $117 \pm 16$ & $196 \pm 16^{\mathrm{A}}$ \\
Vessel diameter $(\mu \mathrm{m})$ & $37 \pm 3$ & $37 \pm 3$ & $20 \pm 2$ & $15 \pm 1^{\mathrm{A}}$ \\
No. of vessel segments $\left(/ \mathrm{mm}^{2}\right)$ & $67 \pm 40$ & $177 \pm 27^{\mathrm{A}}$ & $199 \pm 39$ & $377 \pm 37^{\mathrm{A}}$ \\
\end{tabular}

For tissue NO level measurements, B16F1 and B16F10 tumors in dorsal chambers (59 profiles in 10 tumors and 61 profiles in 9 tumors, respectively) and those in cranial windows (10 profiles in 2 tumors and 15 profiles in 3 tumors, respectively) were examined. For angiogenesis and vessel morphology, 5 random locations per tumor were examined in $3,5,6$, and 5 tumors, from the left to the right columns in the table, respectively. ${ }^{A} P<0.05$ as compared with corresponding B16F1 tumors.

and approximately 20 (17-25) days in the cranial windows and dorsal skin chambers, respectively. There was no significant difference in growth rate in a given site between B16F1 and B16F10 tumors (data not shown). As summarized in Table 1, tissue NO levels paralleled vessel densities among 4 sets of B16 tumors. Both the tissue NO levels and vessel densities in B16F10 tumors were significantly higher than those in B16F1 tumors, regardless of their site of growth. Interestingly, the NO levels and vessel densities were significantly higher in the cranial window tumors than in the dorsal skin chamber tumors in both variants of melanomas. Furthermore, the vascular architecture in B16F10 tumors was remarkably different from that in B16F1 tumors in the cranial window (Supplemental Figure S1; supplemental material available online with

We determined tissue NO level, localization of NOS expression, distribution of NO production, angiogenesis, vessel morphology, and endothelial-mural cell association in murine melanomas to dissect the roles of NO and NOS in morphogenesis and maturation of tumor vessels. Furthermore, we determined the role of NO in mural cell recruitment using independent in vitro assays (28) and a novel in vivo model (29). To dissect the causal relationship, we modified NO production using a pharmacological inhibitor and NOS-deficient mice. We found that (a) NO induces angiogenesis and vessel maturation, specifically vessel branching as well as longitudinal extension and mural cell coverage over tumor vessels in B16 melanomas; (b) in these tumors, eNOS in vascular endothelial cells is the predominant source of NO that mediates angiogenesis, mural cell coverage, and resulting vessel morphology; and (c) endothelial cell-derived NO mediates the directional migration and recruitment of mural cell precursors toward angiogenic vessels both in coculture models in vitro and a tissue-engineered blood vessel model in vivo.

\section{Results}

Tissue NO levels correlate with angiogenic activity in B16 melanomas. Angiogenesis is an essential process for tumor growth and metastasis (30-32). We and others have shown that NO mediates angiogenesis $(4,15-17,33)$. However, the relationship between tissue NO levels and tumor angiogenesis is not known. To clarify this relationship, we determined tissue NO levels and angiogenesis in 2 variants of B16 murine melanomas (the high-metastatic B16F10 and the lowmetastaic B16F1) grown in the primary site (dorsal skin) and a metastatic site (cranium). NO profiles were measured with recessed NO microsensors (34), and microvascular parameters were determined by intravital microscopy (27) when the tumors reached 6-7 $\mathrm{mm}$ in diameter. Tumors reached the desired size in approximately 7 (6-9)

\section{Figure 1}

Effect of chronic NO inhibition on B16F10 tumor vessels in the cranial window. (A) Representative microangiography images of B16F10 melanoma treated with D-NMMA (5 tumors, 5 locations each) or L-NMMA (6 tumors, 5 locations each). Scale bar: $100 \mu \mathrm{m}$. (B-D) Vessel parameters quantified by off-line analyses of the digitized microangiography images. Note that L-NMMA treatment resulted in significantly reduced vascular density (B), larger vessel diameter (C), and reduced branching (D). (E) Histological quantification of endothelial cell nuclei per vessel cross-section in B16F10 melanoma treated with D-NMMA (129 vessels in 3 tumors, 5 locations in each) or L-NMMA ( 83 vessels in 3 tumors, 5 locations in each). ${ }^{*} P<0.05$ as compared with corresponding controls. this article; doi:10.1172/JCI24015DS1). In order to quantify vessel branching, we counted the number of distinct vessel segments in each high-power field. B16F10 tumors had more vessels and branches, while B16F1 tumors had significantly larger but fewer vessels. These data indicate that tissue NO level correlates well with angiogenic activity and metastatic potential of murine melanomas. The data also indicate that host-tumor interaction is an important determinant of angiogenesis in tumors.

NO mediates branching angiogenesis in $B 16$ melanomas. In order to test the causal relationship between NO and angiogenesis, we determined the effect of NOS inhibition on B16F10 tumors grown in the cranial window - the tumors exhibiting highest tissue NO level and angiogenic activity. We used the isoform-nonselective NOS inhibitor $\mathrm{N}^{\mathrm{G}}$-monomethyl-L-arginine monoacetate (L-NMMA), which abolishes NO production of any NOS expressed in the tumor. Chronic L-NMMA treatment resulted in significantly reduced vascular density compared with control $\mathrm{N}^{\mathrm{G}}$-monomethyl-D-arginine monoac-
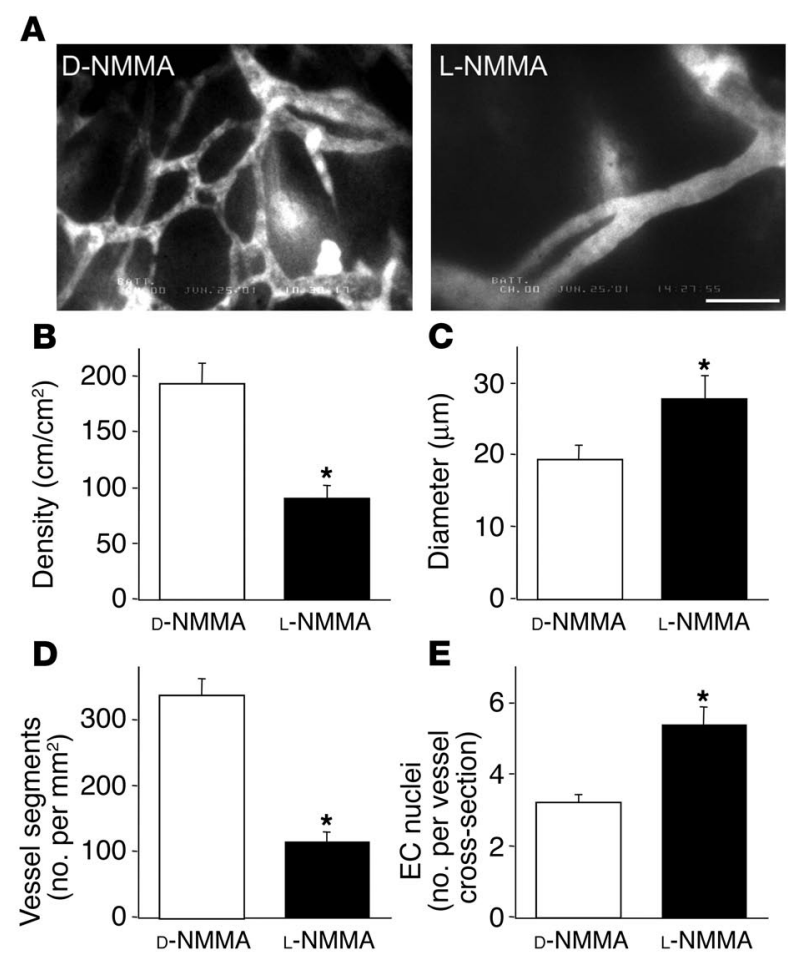
eNOS

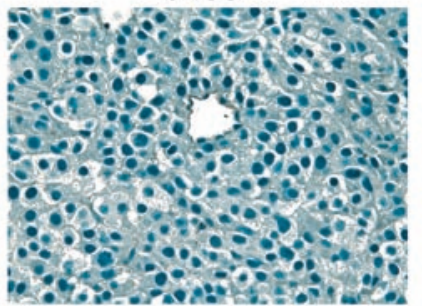

nNOS

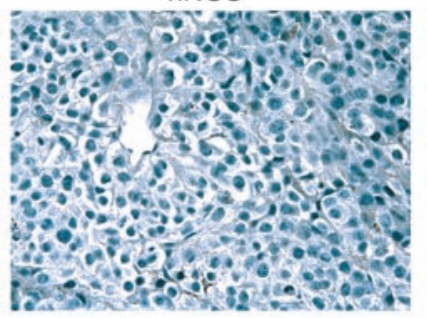

iNOS

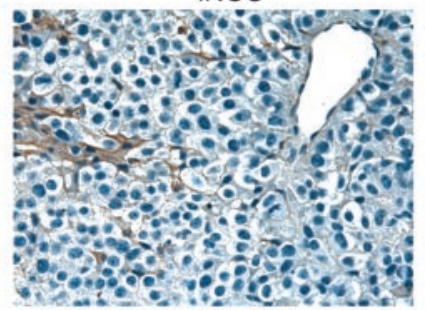

$\lg G$

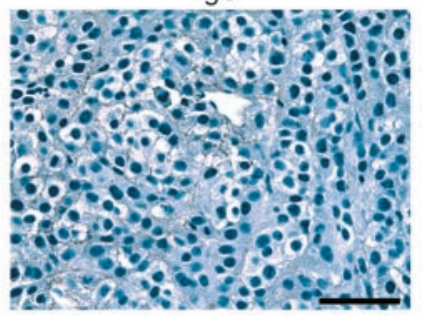

\section{Figure 2}

Immunohistochemical analyses of the expression of NOS isoforms in B16F10 tumors grown in the cranial window. Note the expression of eNOS in endothelial cells (top left) and iNOS in some stromal cells (top right). Also note that there is no expression of nNOS (bottom left). Bottom right panel shows negative control using nonspecific mouse IgG staining. Scale bar: $100 \mu \mathrm{m}$.

etate (D-NMMA) treatment (Figure 1). It is of interest that the vessel morphology of L-NMMA-treated B16F10 tumors is similar to that of B16F1 tumors (Supplemental Figure S1). L-NMMA treatment significantly reduced both vessel density and the number of vessel segments in B16F10 tumors compared with that in D-NMMA-treated tumors (Figure 1D). These data indicate that NO mediates not only the longitudinal extension of blood vessels during angiogenesis but also the development of new branches. These morphological changes in blood vessels were also confirmed by independent histological analyses (Supplemental Table S1). As shown in Figure 1C, average vessel diameter was significantly larger in L-NMMA-treated tumors. Vessel diameter is subject to change by alteration of blood vessel contractile tone and microvascular pressure and thus may not represent circumferential growth of endothelial cells. Therefore, we quantified the number of endothelial cell nuclei per cross-section of each blood vessel in the subsequent histological analyses of B16F10 melanomas treated with D-NMMA and L-NMMA. In agreement with intravital microscopy and histological observation, we found a significantly larger number of nuclei per vessel cross-section in L-NMMA-treated tumors than in the control tumors (Figure 1E). These results indicate that the larger diameter of L-NMMA-treated tumors is attributable to the proliferation of endothelial cells. Considering these data collectively, we conclude that NO promotes vessel branching and longitudinal extension but not the luminal growth of blood vessels in B16 melanomas.

B16 melanomas express vascular eNOS and $i N O S$ sporadically in stroma but do not significantly express $n N O S$. To identify which NOSs and cells are responsible for NO production, we first determined the localization of NOS in B16F10 tumors grown in the cranial windows (Figure 2). Immunohistochemical observation for the 3 isoforms of NOS revealed relatively homogeneous expression of eNOS in vascular endothelial cells in both normal brain tissue (not shown) and tumors. On the other hand, iNOS expression was found sporadically in some host stromal cells. Although we found that neurons in normal brain tissue were positive for nNOS (not shown), nNOS was hardly detectable in the B16F10 tumors. It appeared that B16 tumor cells do not appreciably express NOSs.

NO is mainly distributed at the blood vessel wall in B16 melanomas. The presence of NOS does not necessarily indicate the production of NO since the activity of NOS is regulated by various factors such as intracellular calcium/calmodulin, heat shock protein 90, subcellular

\section{Figure 3}

Distribution of NO in B16F10 tumors grown in the cranial window and the effect of chronic $\mathrm{NO}$ inhibition. The animals were treated with D-NMMA (A-C) or L-NMMA (D-F). (A, D, and G) Microangiography using tetramethylrhodaminedextran $\left(2 \times 10^{6}\right.$ molecular weight). (B and $\left.\mathbf{E}\right)$ Representative microfluorography captured 60 minutes after the loading of DAF-2 in tumors. (C and F) Pseudocolor representation of DAF-2T microfluorographs. Color bar in the top right shows calibration of the fluorescence intensity with known concentrations of DAF-2T. (G-I) Control tumor (no treatment). (G) Microangiograph. (H) Microfluorograph captured at 60 minutes after the injection of 4-AF, an NO-insensitive control fluorochrome, and its pseudocolor representation (I). Color bar in the bottom-right portion shows calibration of fluorescence intensity with known concentrations of 4-AF. Scale bar: $100 \mu \mathrm{m}$.
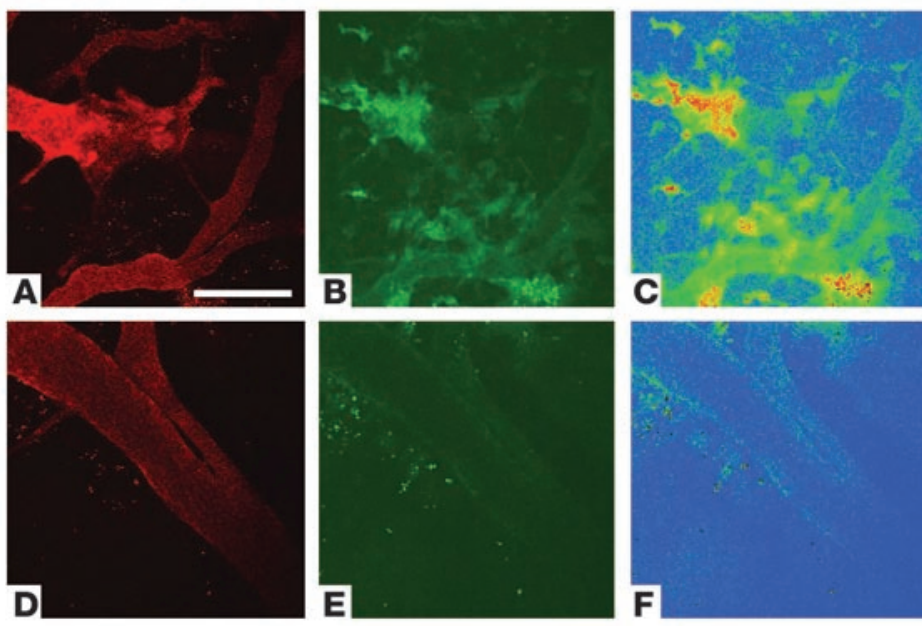

[DAF-2T] $(\mathrm{nmol} / \mathrm{l})$
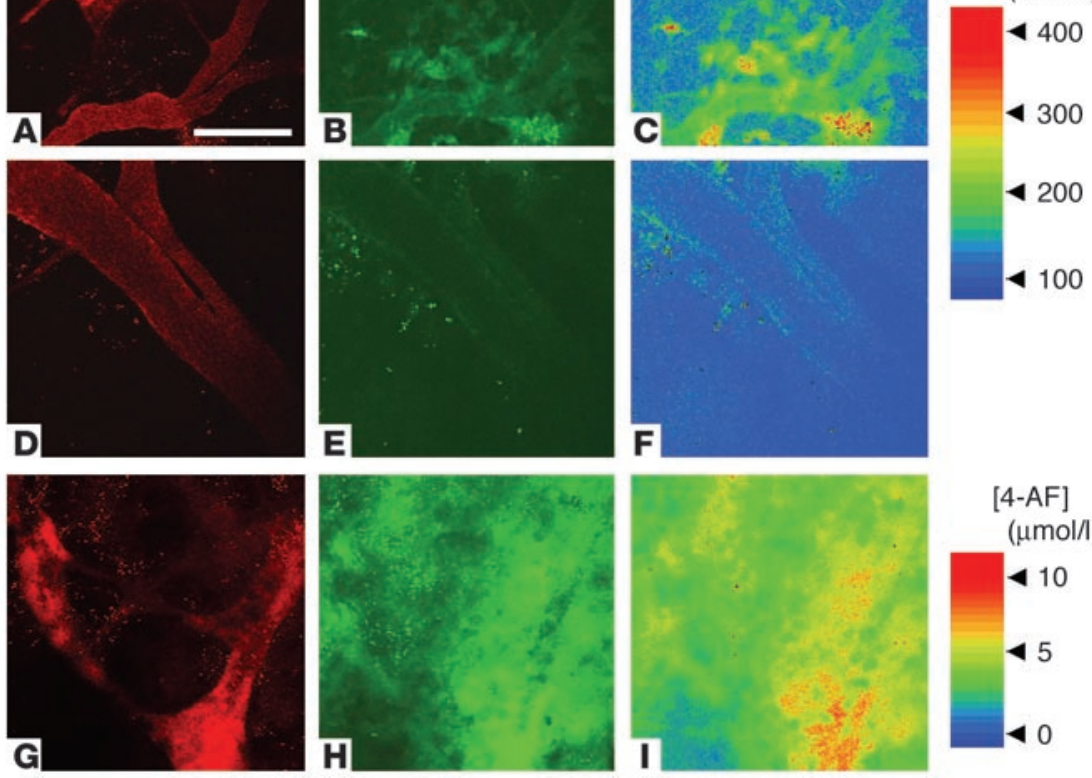
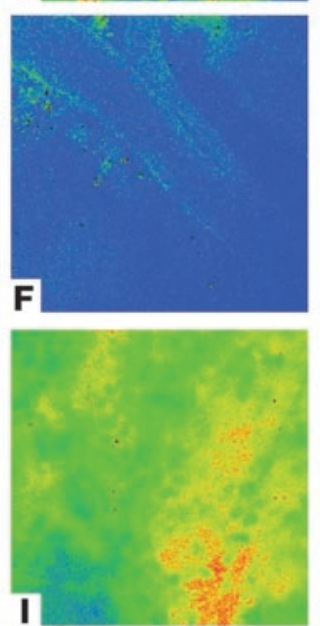

[4-AF]

$(\mu \mathrm{mol} / \mathrm{l})$

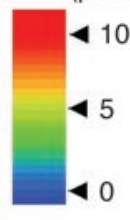


A

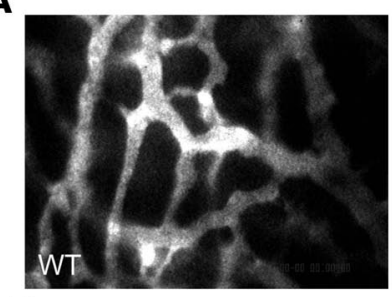

B
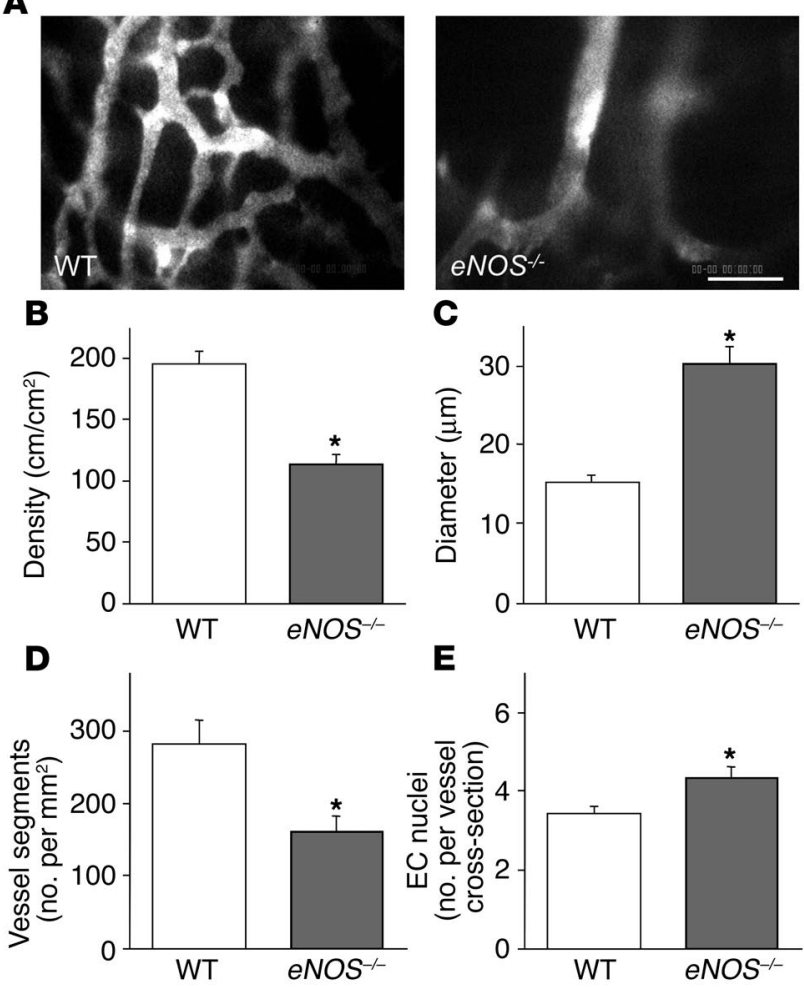

C

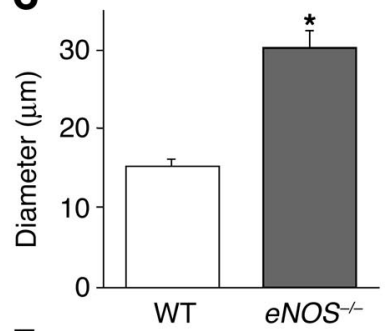

E

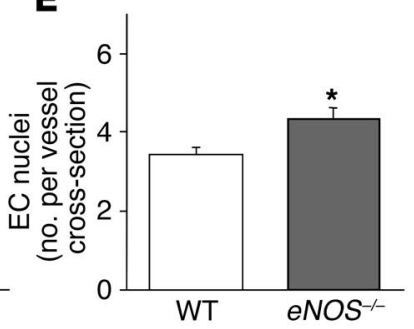

localization, interaction with caveolin, phosphorylation, protein stability, dimerization, cofactor binding, and availability of oxygen and L-arginine $(35,36)$. Therefore, we visualized NO distribution in vivo using an NO-sensitive fluorescent probe 4,5-diaminofluorescein (DAF-2). NO converts DAF-2 to DAF-2 triazole (DAF-2T) in the presence of oxygen. DAF-2T is 180 times more fluorescent than DAF-2 (37). We used multiphoton laser-scanning microscopy (MPLSM) to clearly visualize the subcellular localization of the fluorescent probe. In B16F10 tumors, DAF-associated fluorescence increased with time after loading and was found around the blood vessel wall and sporadically in the extravascular space (Figure 3). DAF-associated fluorescence in the vessel wall and extravascular space was abolished by the administration of L-NMMA (Figure 3). On the other hand, fluorescence of 4-aminofluorescein (4-AF), an NO-insensitive but structurally similar probe (38), was distributed homogeneously and showed no significant changes after loading (Figure 3). According to a previous report, $\mathrm{NO}$ signal is primarily confined to within $10 \mu \mathrm{m}$ of the source (39); thus, the origin of $\mathrm{NO}$ in the vessel wall and interstitium should be distinct. Taken together with our immunohistochemical analyses, these results suggest that $\mathrm{NO}$ is produced predominantly by eNOS in vascular endothelial cells and only sporadically by iNOS in the stromal cells of B16 melanomas.

Host cell eNOS but not iNOS contributes to angiogenesis and vessel morphogenesis in 1616 melanomas. To determine the contribution of host cell eNOS- and iNOS-derived NO to tumor angiogenesis and vessel morphology, we performed intravital microscopy of B16F10 tumors in eNOS - ,, $\mathrm{NOS}^{-/}$, and wild-type C57BL/6 mice. Vessel density was significantly reduced but diameter was significantly larger in $e \mathrm{NOS}^{-/-}$compared with wild-type mice (Figure 4). As shown in Figure 4A, vessel morphology in $e \mathrm{NOS}^{-/-}$mice was similar to that in L-NMMA-treated tumors (Figure 1A) and B16F1 tumors (Supplemental Figure S1). There was significantly less branching in $\mathrm{eNOS}^{-/}$mice

\section{Figure 4}

Effect of host eNOS deletion on B16F10 tumor vessels in the cranial window. (A) Representative microangiography images of B16F10 melanoma grown in wild-type C57BL/6 or eNOS ${ }^{--}$mice. Scale bar: $100 \mu \mathrm{m}$. (B-D) Vessel parameters quantified by off-line analyses of the digitized microangiography images. Note that B16F10 tumors in eNOS ${ }^{-/}$mice $(n=5)$ have significantly reduced vascular density $(\mathbf{B})$, larger vessel diameter (C), and reduced branching (D) as compared with those in C57BL/6 mice $(n=5)$. (E) Histological quantification of endothelial cell nuclei per vessel cross-section in B16F10 tumors grown in C57BL/6 (132 vessels in 3 tumors, 5 locations in each) or eNOS ${ }^{-/-}$mice (92 vessels in 3 tumors, 5 locations in each). ${ }^{\star} P<0.05$ as compared with corresponding controls.

than in wild-type mice (Figure 4D). Histological analyses confirmed intravital microscopy observations that revealed B16 tumors grown in $\mathrm{eNOS}^{-/}$mice had decreased vessel numbers but increased vessel perimeters and numbers of endothelial nuclei per vessel cross-section compared with wild-type mice (Supplemental Table S1; Figure 4E). In contrast, the deletion of $i N O S$ in the host stromal cells did not significantly affect angiogenesis and vessel morphology of B16F10 melanomas (Supplemental Figure S2). Although analyses of spatial and temporal distribution of NO by DAF-2 microfluorography showed that both $\mathrm{eNOS}$ and iNOS produce NO in B16 melanomas (Figures 2 and 3), eNOS appeared to play a predominant role in NO-induced tumor angiogenesis. Taken together, these data suggest that $\mathrm{NO}$ produced by eNOS in the host endothelial cells governs the branching and longitudinal extension of blood vessels in B16 melanomas.

$N O$ from eNOS regulates the extent of mural cell coverage of B16 melanoma vessels. The association of pericytes with vascular endothelial cells has been suggested as regulating endothelial cell proliferation, survival, migration, and differentiation $(21,23,40)$. To understand the role of $\mathrm{NO}$ in the association of mural cells with tumor vessels and the relationship between mural cell coverage and tumor-vessel architecture, we determined mural cell coverage by immunohistochemistry in tumors with and without modification of $\mathrm{NO}$ production. The mural and endothelial cells were identified in serial sections of a histological specimen by antibodies specific for mural cell markers, $\alpha$-SMA, and chondroitin sulfate proteoglycan 4 (NG2) as well as a mouse endothelial cell-specific differentiation antigen (MECA-32) (Figure 5). The 2 mural cell markers had essentially identical distributions and colocalized at least in part with most blood vessels in both B16F10 (Figure 5A) and B16F1 (not shown) melanomas, although the sensitivity of NG2-antibody staining appeared to be lower than that of $\alpha$-SMA staining. The extent of the blood vessel coverage of $\alpha$-SMA- or NG2-positive mural cells was analyzed using NIH Image 1.63 freeware. Mural cell coverage was significantly lower in B16F1 tumors than in B16F10 tumors (Figure 6). This paralleled the difference in tissue $\mathrm{NO}$ level and vessel density, but an inverse relationship was found between mural cell coverage and average vessel diameter (Table 1). Next, we compared mural cell coverage of B16F10 tumor vessels under chronic NO inhibition (L-NMMA treatment) with that in the control tumors. Mural cell coverage in B16F10 tumors was significantly decreased by L-NMMA treatment (Figures 5B and 6). These results suggest that $\mathrm{NO}$ regulates the extent of mural cell coverage of tumor vessels in B16 melanomas. Further, to reveal the relative contribution of each isoform of NOS, we compared mural cell recruitment in $\mathrm{eNOS}^{-/}$and $i \mathrm{NOS}^{-/}$mice with that in wild-type mice. The extent of mural cell coverage was significantly less in B16F10 tumors grown in eNOS ${ }^{-/-}$mice, but the extent observed in $i \mathrm{NOS}^{-/-}$ mice was comparable to that in wild-type mice (Figures 5B and 6). 
A

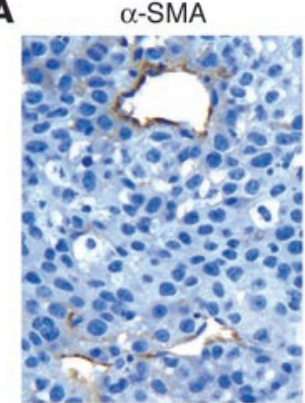

B

D-NMMA
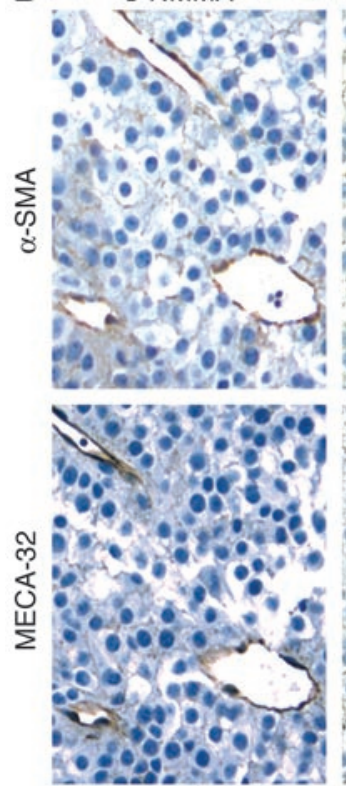

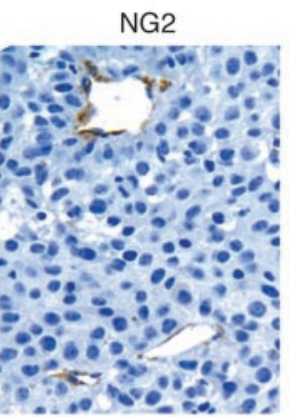

L-NMMA
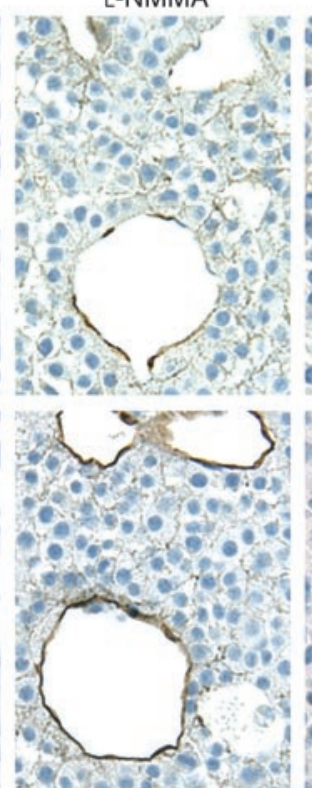

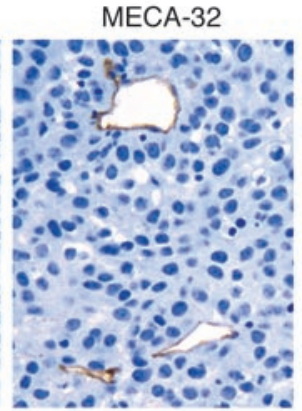

WT
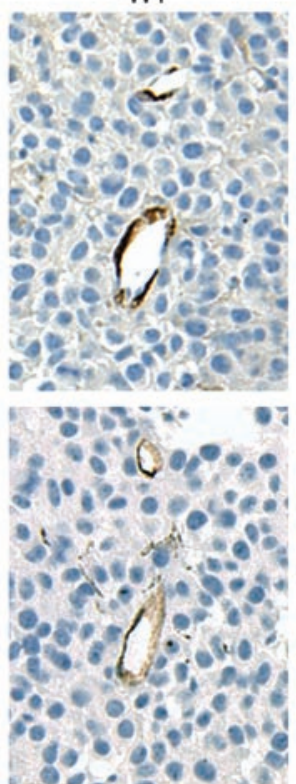

Figure 5

Immunohistochemical observation of mural cell coverage in B16F10 tumors grown in the cranial window. Five micron paraffin block sections were immunostained using the avidin-biotin complex/ diaminobenzidine histochemistry method. (A) Control B16F10 tumor sections were stained for mural cells using monoclonal mouse anti- $\alpha$-SMA or polyclonal rabbit anti-NG2 and endothelial cells using polyclonal rat anti-MECA-32. Two markers for mural cells showed essentially identical distributions. (B) B16F10 tumors treated with D-NMMA or L-NMMA or grown in wild-type C57BL/6 or eNOS ${ }^{-/}$mice. Scale bars: $100 \mu \mathrm{m}$.

Taken together, these results indicate that $\mathrm{NO}$ regulates mural cell coverage of B16 melanoma vessels and that NO produced by eNOS predominantly mediates this process.

NO suppression did not alter endogenous angiogenic gene expression in $B 16$ melanomas. To investigate the possible involvement of endogenous angiogenic factors in NO-induced mural cell coverage and vessel morphogenesis, we determined the expression profile of 96 murine genes associated with angiogenesis in B16F10 melanoma with and without NO inhibition using a cDNA microarray. Although expression of a number of genes was detected in B16F10 tumors ( 90 out of 96 were expressed), none of the genes in L-NMMA-treated tumors were differentially expressed by more than 2 -fold compared with those in D-NMMA-treated tumors (Supplemental Table S2). Specifically, the expression of several genes that mediate angiogenesis and vessel maturation (PDGF-B, PDGFR $\beta$, angiopoietin- 1 and -2 , Tie-2, sphingosine kinase type 1 , endothelial differentiation sphingolipid G-protein-coupled receptor 1 (EDG-1), VEGF-A, VEGFR-1, and VEGFR-2) was not significantly different between

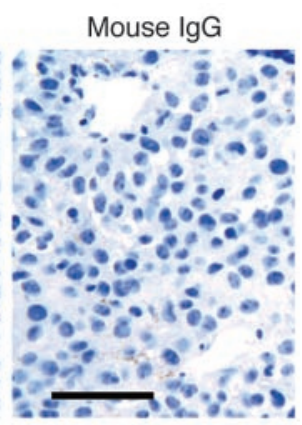

eNOS-1-
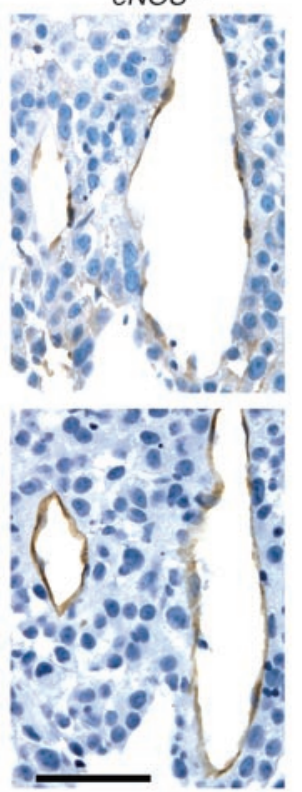

L-NMMA- and D-NMMA-treated B16F10 tumors, as shown by quantitative real-time PCR (Supplemental Table S3). Thus, it is not likely that NO regulates mural cell coverage and vessel morphogenesis via transcriptional regulation of these known angiogenic and vessel maturation factors.

NO from endothelial cells mediates migration of mural cell precursor. In order to determine the role of endothelial NO in mural cell recruitment, we performed 2 different in vitro migration assays. The migration of $10 \mathrm{~T} 1 / 2$ cells, mural cell precursors, through transwell filters or under the agarose gel toward human umbilical vein endothelial cells (HUVECs) was quantified with or without NOS inhibition. As shown in Figure 7, robust transwell migration of 10T1/2 cells was observed in the presence of HUVECs, and application of $100 \mu \mathrm{M}$ L-NMMA significantly reduced this migration. To examine directional migration, HUVECs and 10T1/2 cells were cultured $2.5 \mathrm{~mm}$ apart in the under-agarose assay. 10T1/2 cells migrated toward the HUVECs in a directional manner (Figure 8). L-NMMA treatment decreased 10T1/2 cells' migration toward HUVECs, both in number and distance (Figure 8 and Table 2). To evaluate the possible contribution of $10 \mathrm{~T} 1 / 2$ cell proliferation in the under-agarose assay, we examined proliferating cell nuclear antigen (PCNA) staining. The PCNA-positive rate in $10 \mathrm{~T} 1 / 2$ cells was significantly higher in the L-NMMA-treated group than in the control group (Table 2). We therefore conclude that an increase in 10T1/2 cell numbers located at the side of HUVECs is due to directional cell migration, not cell proliferation. Taken together, these results suggest that $\mathrm{NO}$ derived from endothelial cells induces directional migration of mural cell precursors.

NO mediates recruitment of mural cells in vivo. We next examine whether NO is involved in the recruitment of mural cells to angiogenic vessels in vivo. To this end, we used a tissue-engineered blood vessel model in which HUVECs and 10T1/2 cells embedded in a collagen-fibronectin gel were implanted in the mouse cranial window to form stable and functional blood vessels (29). HUVECs and 10T1/2 cells were transfected with enhanced GFP (eGFP) and red fluorescent protein from Discosoma (DsRed) genes, respectively, then visualized by MPLSM, and their interaction was analyzed by an NIH Image 1.63 freeware macro (Figure 9) $(26,29)$. After the implantation, 10T1/2 cells started to interact with HUVECs; the coverage of 10T1/2 cells around the network-forming HUVECs increased in a time-dependent manner up to 21 days and thereafter became stable (Figure 10). Following 10T1/2 cell recruitment, 

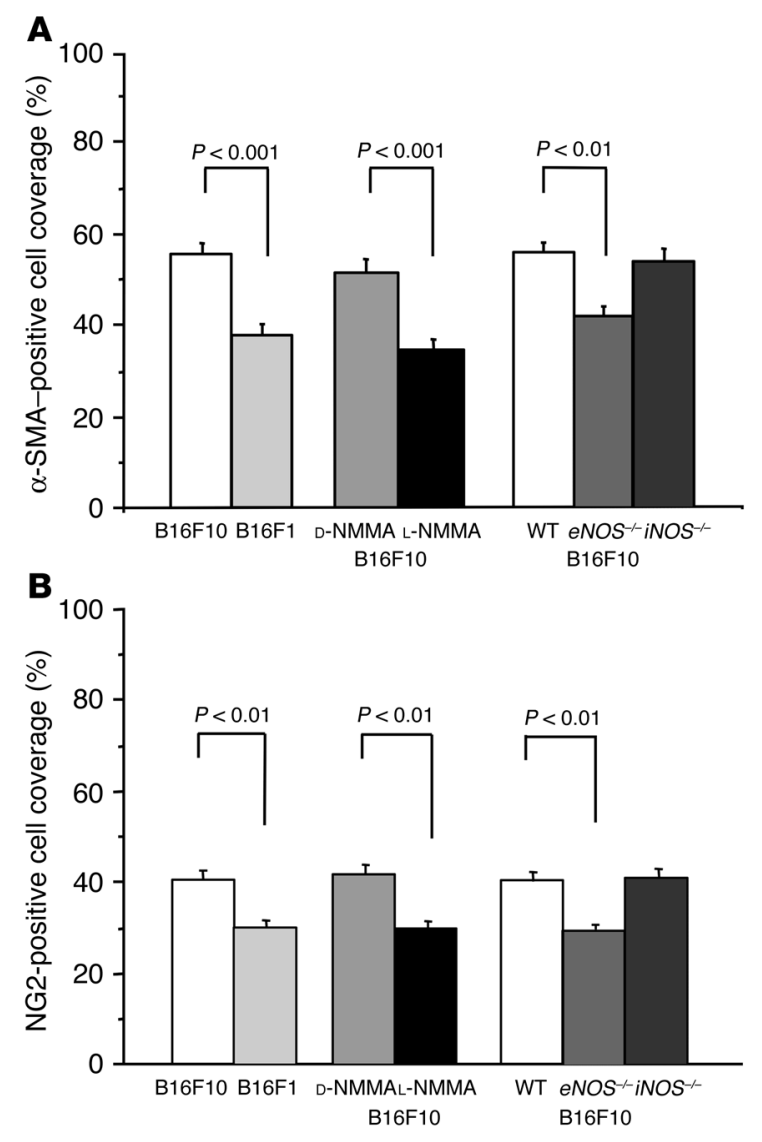

the perfusion of the HUVECs network began, increased with time, and became stable after 21 days. L-NMMA treatment significantly inhibited both the early interaction of $10 \mathrm{~T} 1 / 2$ cells and HUVECs before perfusion as well as the recruitment of $10 \mathrm{~T} 1 / 2$ cells to the perfused HUVECs network (Figure 10). Furthermore, the perfused vascular density was significantly lower, and the total vessel density was decreased with time in the L-NMMA-treated group (Figure 10). We also determined the morphology of the tissue-engineered blood vessels. L-NMMA treatment significantly decreased both the length and the extent of branching of the vessels but did not affect vessel diameter (Table 3 ). As reported previously (29), HUVECs implanted alone (without $10 \mathrm{~T} 1 / 2$ cells) show only transient vessel formation and perfusion that is significantly less than that seen in HUVEC-10T1/2 coimplantation. L-NMMA did not affect these non-mural cell-mediated processes. Taken together, these results indicate that NO suppression impairs

\section{Figure 7}

Transwell migration of $10 \mathrm{~T} 1 / 2$ cells toward HUVECs. (A) Transmigrated eGFP-10T1/2 cells at the back side of the FluoroBlok insert. Scale bar: $200 \mu \mathrm{m}$. (B) Percentage area of the transwell filter covered by migrated 10T1/2 cells. Medium only, no HUVECs in the bottom well. ${ }^{*} P<0.05$ as compared with the control group. All transwell migration assays were conducted in triplicate, and each set of experiments was performed 3 times.

\section{Figure 6}

Extent of mural cell coverage in B16 melanomas grown in the cranial window. Percentage coverage of blood vessels by $\alpha$-SMA-positive cells (A) and NG2-positive cells (B) was determined. Note that the extent of pericyte coverage was significantly lower in B16F1 or in L-NMMA-treated B16F10 tumors than in control B16F10 tumors. B16F10 tumors grown in eNOS ${ }^{-/-}$but not in NOS $^{-/-}$mice showed smaller coverage than those in wild-type mice. Three tumors of 5 locations each were determined for all groups. The numbers of vessels determined (from left to right) are 127, 91, 109, 77, 127, 105, 114 (A) and 144, 83, 138, 84, 114, 107, 147 (B).

mural cell recruitment and the subsequent establishment and remodeling of a stable functional vessel network.

\section{Discussion}

This study shows tissue NO levels and spatial distribution of NO in tumors using NO-sensitive microelectrodes and fluorescent probes, respectively. We found that the average tissue NO levels in B16 melanomas were 1.5-3.1 $\mu \mathrm{M}$, depending on the tumor cell variant and site of tumor growth, and that tissue NO levels correlated well with angiogenic activity (Table 1). Tumor-tissue NO values are higher than those in normal quiescent tissue. For example, the NO level in the cortex of SCID mice is approximately $0.7 \mu \mathrm{M}$ (our unpublished data). High NO levels $(\sim 2.0 \mu \mathrm{M})$ in angiogenic tissues have been reported with a collagen gel implant in which angiogenesis is induced by recombinant VEGF (17). Inhibition of $\mathrm{NO}$ production resulted in reduced angiogenesis in this model as is also seen in the current study. Collectively, these data indicate that NO induces angiogenesis in tumors.

Imaging of DAF-2, an NO-sensitive probe, revealed the production of $\mathrm{NO}$ at the blood vessel wall in B16 melanomas (Figure 3). Immunohistochemistry indicated that eNOS in vascular endothelial cells is responsible for NO production at the vasculature. In nontumor-bearing cranial windows, DAF-2 fluorescence also clearly localized at the vasculature (Supplemental Figure S3). Of interest, DAF-2 fluorescence was stronger in arterioles than in capillaries and venules in normal pial vessels. This finding is consistent with a previous report of strong arteriolar NO production in rat mesentery where $\mathrm{nNOS}$ expression in periarteriolar nerves was observed in addition to eNOS expression in vascular endothelial cells (41). On the other hand, we could not find
A

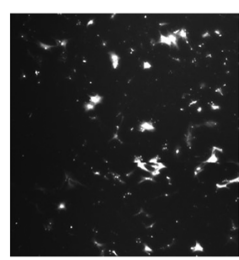

Control

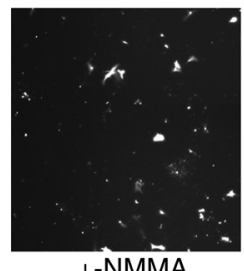

L-NMMA

$10 \mu \mathrm{M}$

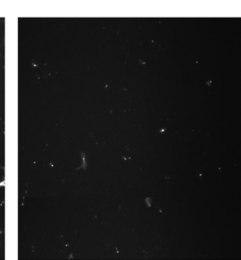

Medium only

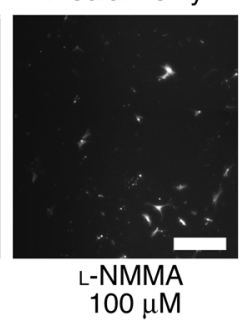

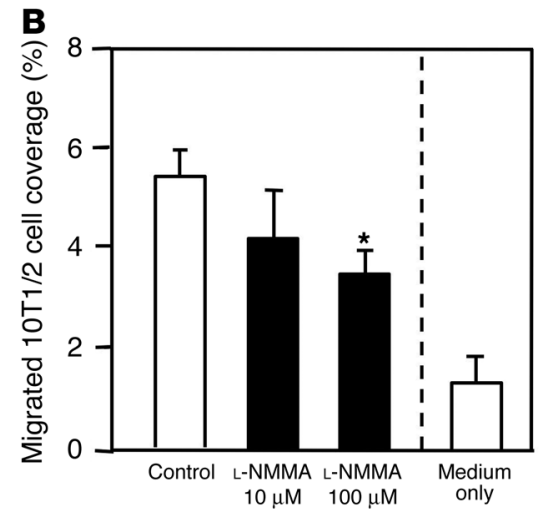


A

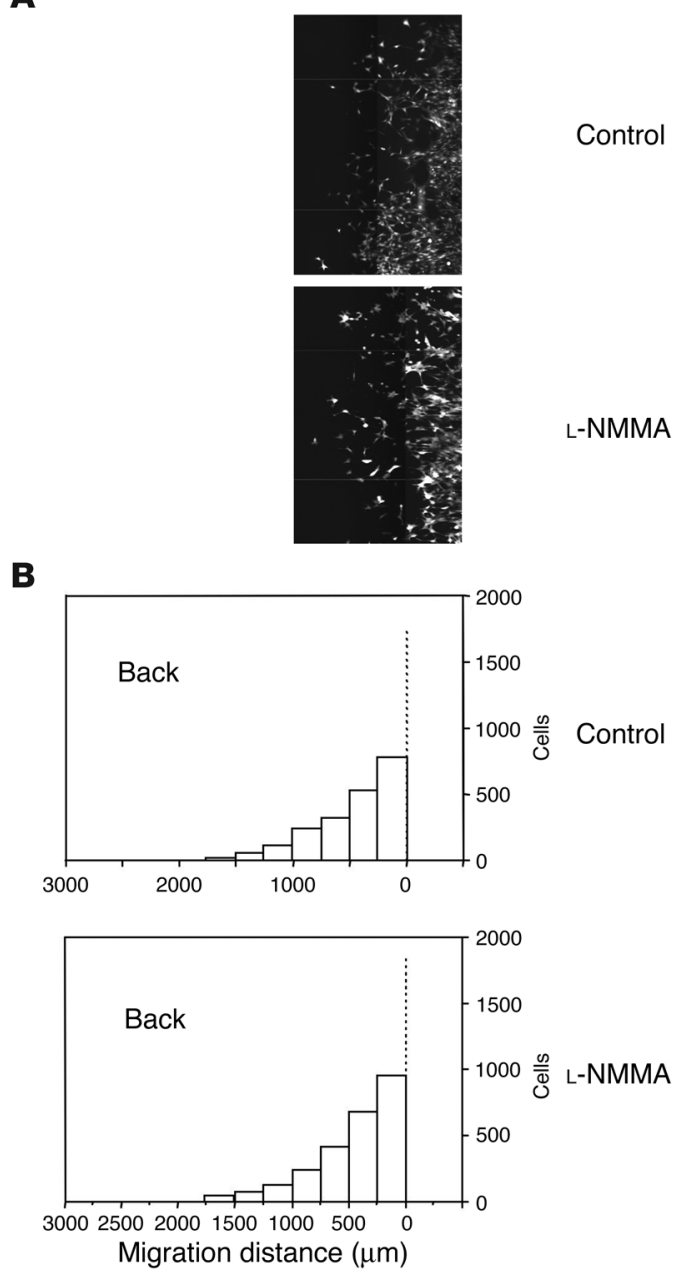

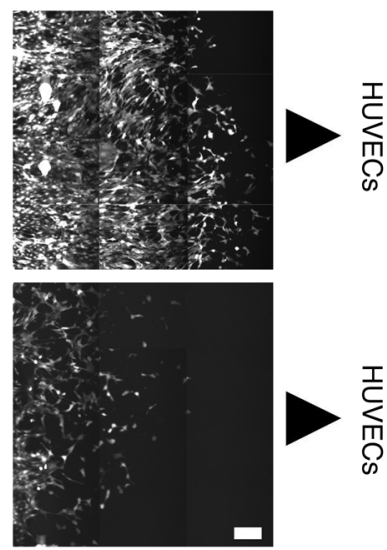
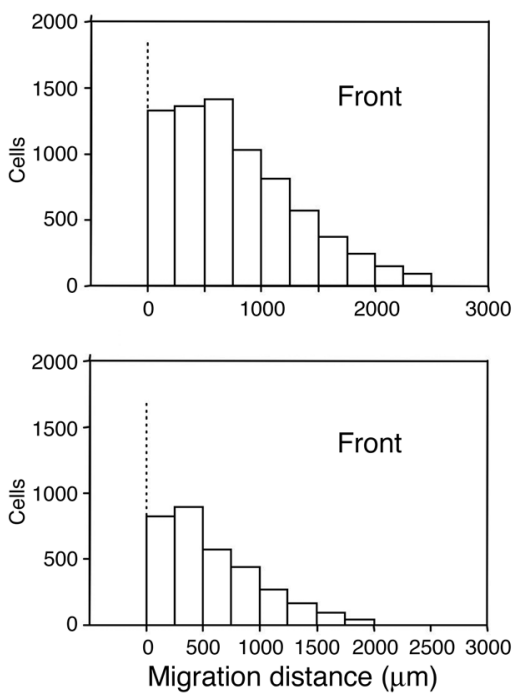

Figure 8

Under-agarose 10T1/2 cell migration in the presence of HUVECs. (A) Representative images of migrated eGFP-10T1/2 cells in the control (top row) and L-NMMA-treated (100 $\mu \mathrm{M}$; bottom row) groups. HUVECs are located on the right-hand side of the images. Representative images of a part of the agarose well both in the direction to HUVECs (front; right panels) and in the opposite direction to HUVECs (back; left panels) are shown. Scale bars: 200 um. (B) Histograms of the migrated 10T1/2 cells. All under-agarose migration assays were conducted in duplicate, and each set of experiments was performed 3 times. Histograms of the sum of all 6 independent experiments are shown.

grown in iNOS-deficient mice $(19,20)$. However, sporadic expression of iNOS in stromal cells did not significantly affect angiogenesis, vessel morphology, and mural cell recruitment in B16 melanomas grown in cranial windows in this study. The cranium is known to be an immunoprivileged site. In fact, we did not find an appreciable inflammatory cell accumulation in tumors grown in cranial windows or a difference in angiogenesis in angiogenic gel assays of immunocompetent and immunodeficient mice with the same background (17). This may explain the insignificant effect of host iNOS deletion in our study. The cranial window model used in this study allowed us to clearly determine the role of vascular endothelial eNOS on tumor angiogenesis. It is noteworthy that iNOS has been reported to mediate the induction of VEGF in the above-mentioned studies $(19,20,43)$ and that eNOS predominantly mediates VEGF-induced angiogenesis (17). NO suppression did not alter endogenous VEGF expression in this study (Supplemental Table S3).

Although several reports of both clinical and experimental tumors have shown that NO plays an important role in tumor angiogenesis (12-14), there is no study showing how $\mathrm{NO}$ affects vessel morphology in tumors. NO has been shown to mediate cell migration and proliferation as well as tube formation and survival of endothelial cells in vitro $(33,44)$. In this study, we found that NO mediates branching and longitudinal extension of blood vessels in B16 melanomas (Figure 1, Supplemental Table S1) and that this process is predominantly mediated by eNOS (Figure 4 and Supplemental Table S1). If chronic inhiappreciable nNOS expression in B16 melanomas (Figure 2). These results suggest that tumor vessels do not have nNOS-expressing nerves along with them. Tumor vessels are well known as having structural and functional abnormalities (42). In fact, most tumor vessels remain immature and will not differentiate into functional units such as arterioles, capillaries, and venules. Lack of arteriolar differentiation and innervations with nNOS-positive nerves may also explain the lack of neuronal control of blood flow in tumors.

In addition to vascular NOS, expression of NOS in tumor cells has been reported in various tumors including nNOS in CNS tumors and $\mathrm{iNOS}$ and eNOS in various tumor types (reviewed in ref. 4). Nonvascular sources of NO may coordinate angiogenesis and vessel remodeling mediated by vascular endothelial cell-derived NO. Transduction of the iNOS gene into p 53 mutant tumor cells has been shown to increase neovascularization (43). Reduced angiogenesis and tumor growth were observed in lung and subcutaneous tumors bition of NO suppresses tumor angiogenesis because of the inhibition of endothelial cell proliferation, then vessel diameter and the density of endothelial cell nuclei should also be decreased. However, average vessel diameter was larger in tumors with lowered NO production than in corresponding controls. Circumferential growth of blood vessels was unaltered by $\mathrm{NO}$ inhibition presumably because of other endothelial growth stimuli present in the tumors such as VEGF (Supplemental Tables S2 and S3). In fact, we did not observe a difference in vessel diameter in the tissue-engineered blood vessels with L-NMMA treatment even though this treatment inhibited branching and longitudinal extension of blood vessels (Table 3).

In addition to the direct effect of $\mathrm{NO}$ on endothelial cells or the indirect effect on endogenous angiogenic factors, other mechanisms should be considered in order to understand the vascular morphology observed in B16 melanomas with different NO levels. Blood vessels consist of 2 different types of cells, vascular endothelial cells 


\section{Table 2}

Quantification of 10T1/2 cell migration and proliferation in under-agarose migration assay

\begin{tabular}{lcccc} 
& \multicolumn{2}{c}{ Control } & \multicolumn{2}{c}{ L-NMMA } \\
& Front & Back & Front & Back \\
Migration distance $(\mu \mathrm{m})$ & $618 \pm 56$ & $300 \pm 40$ & $412 \pm 67^{\mathrm{A}}$ & $361 \pm 52$ \\
No. of migrated cells $($ counts/well) & $1161 \pm 212$ & $519 \pm 139$ & $544 \pm 265^{\mathrm{A}}$ & $487 \pm 70$ \\
PCNA positive rate $(\%)$ & $12 \pm 2$ & $14 \pm 4$ & $30 \pm 5^{\mathrm{A}}$ & $28 \pm 5^{\mathrm{A}}$
\end{tabular}

All under-agarose migration assays and PCNA staining were conducted in duplicate, and each set of experiments was performed 3 times. Migration distance was derived from mean of the median value of each experiments. ${ }^{A} P<0.05$ as compared with corresponding controls. tal Figure S4). On the other hand, 2 different types of in vitro migration assays showed that HUVECs recruit 10T1/2 cells via NO (Figures 7 and 8 , and Table 2). Furthermore, 10T1/2 cell proliferation appeared to be inhibited by $\mathrm{NO}$ when these cells were cocultured with HUVECs (Table 2). A coculture system with endothelial cells and murine embryonic mesenchymal cells (10T1/2 cells) was employed as a model of mural cell recruitment in vitro and showed involvement of PDGF and TGF- $\beta 1$ in the recruitment and differentiation of $10 \mathrm{~T} 1 / 2$ cells, respectively (28). However, microarray analysis of angiogenic genes in HUVECs did not show significant alteration with and mural cells (22). The heterotypic cell-cell interaction in the vessel wall affects both cell populations and is suggested as regulating formation, remodeling, stability, and function of blood vessels (21-23). Pericytes may play a role in endothelial sprouting and in vascular branching morphogenesis because of their localization at capillary branch points (23). Furthermore, pericyte sleeves, which are longer than endothelial cell sprouts, have been found. This finding suggests the possibility that they guide and/or regulate sprout growth (45). The lack of pericyte coverage may abrogate microvessel architecture since pericytes stabilize vascular endothelial cells and negatively regulate their proliferation (46). For example, pericytes became detached during endothelial cell hyperproliferation induced by ectopic expression of VEGF-A in normal tissue (47). Here we found that chronic inhibition of NO significantly decreased mural cell association with tumor vasculature and resulted in the increased vessel diameter and decreased vessel density and that eNOS mediated these processes (Figure 6). Proper incorporation of mural cells to the vessel wall structure, including appropriate number, phenotype, and alignment, is an important step of vessel maturation. Multiple molecular mechanisms have been reported as mediating vessel maturation (reviewed in ref. 22). Several key signaling pathways are involved in mural cell recruitment, including PDGF/PDGFR $\beta$, sphingosine-1-phosphate/EDG-1, and angiopoietin-1/Tie-2 (48-50). However, our gene expression analyses did not suggest the involvement of transcriptional regulation of these factors in NO-induced vascular morphogenesis (Supplemental Table S3).

Although there are conflicting reports on the effect of exogenous NO on cultured vascular SMC proliferation and migration $(51,52)$, the role of $\mathrm{NO}$ in mural cell recruitment has yet to be documented. Therefore, we performed a series of in vitro and in vivo analyses without involving cancer cells. First, we checked the direct effect of exogenous $\mathrm{NO}$ on $10 \mathrm{~T} 1 / 2$ cell proliferation. An NO donor, (Z)-1-[2-(2-Aminoethyl)-N-(2-ammo nioethyl)amino]diazen-1-ium-1,2-diolate (DETA NONOate, Alexis Corp.) inhibited 10T1/2 cell proliferation at high (>1 mM) doses (Supplemen-

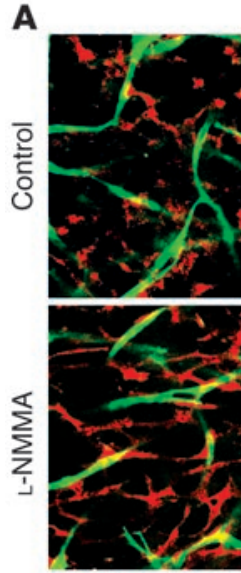

4 days

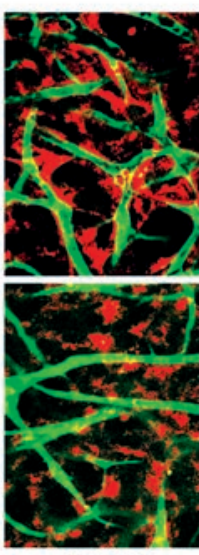

7 days

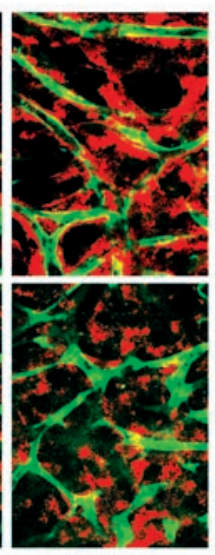

10 days

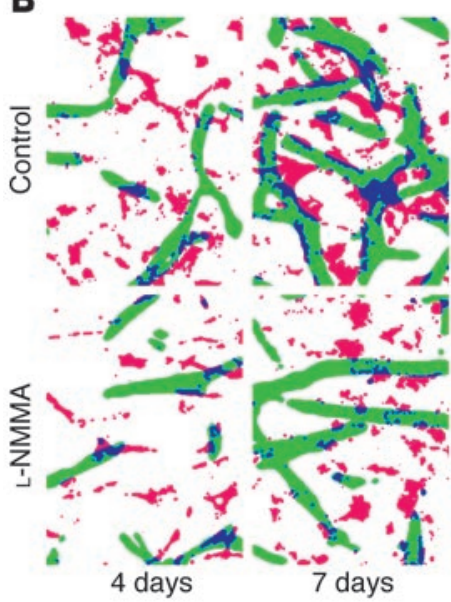

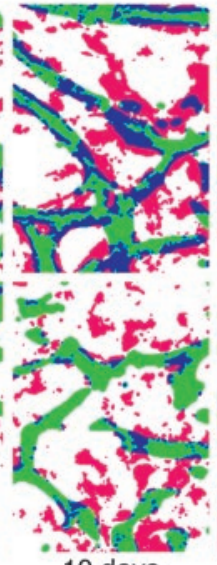

10 days

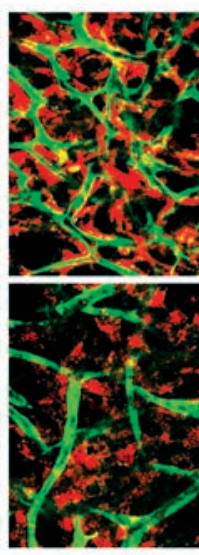

14 days

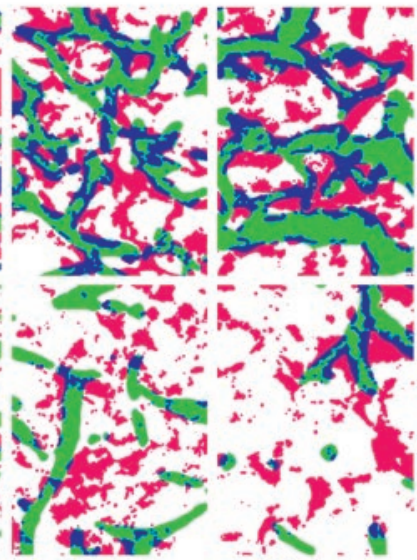

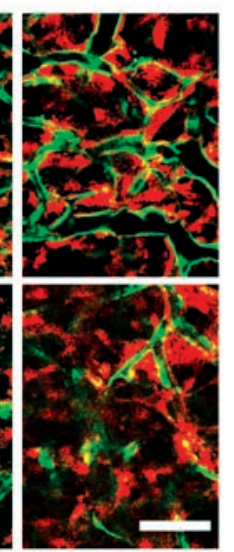

28 days

\section{Figure 9}

Endothelial-mural cell interaction in the tissue-engineered blood vessels. (A) Representative MPLSM images of eGFP-HUVECs (green) and DsRed-10T1/2 cells (red) at 4, 7, 10,14 , and 28 days after implantation with and without L-NMMA ( $7 \mathrm{mg} /$ day) treatment. (B) Analysis of the endothelial-mural cell interaction. Images of endothelial cells (green) and 10T1/2 cells (red) were binarized. The diameters of binarized HUVECs areas were dilated by $4 \mu \mathrm{m}$ from the original edges. Overlap of the 2 cell populations (coverage of mural cells) is shown in blue. Scale bars: $100 \mu \mathrm{m}$. 


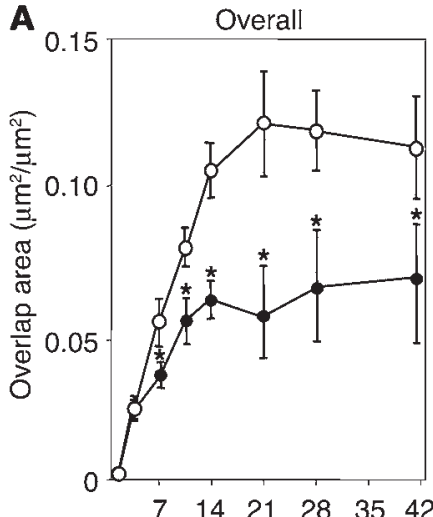

Days after implantation

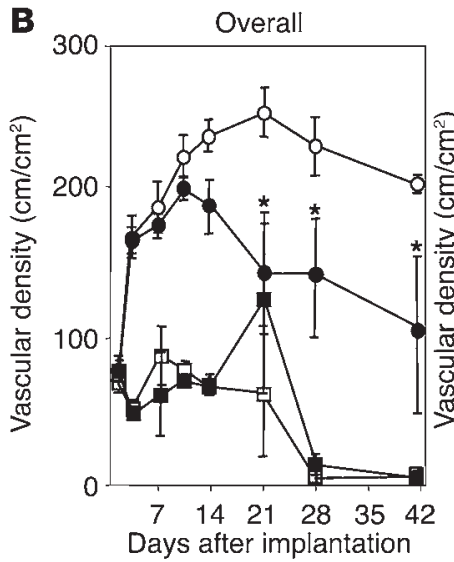

Days after implantation
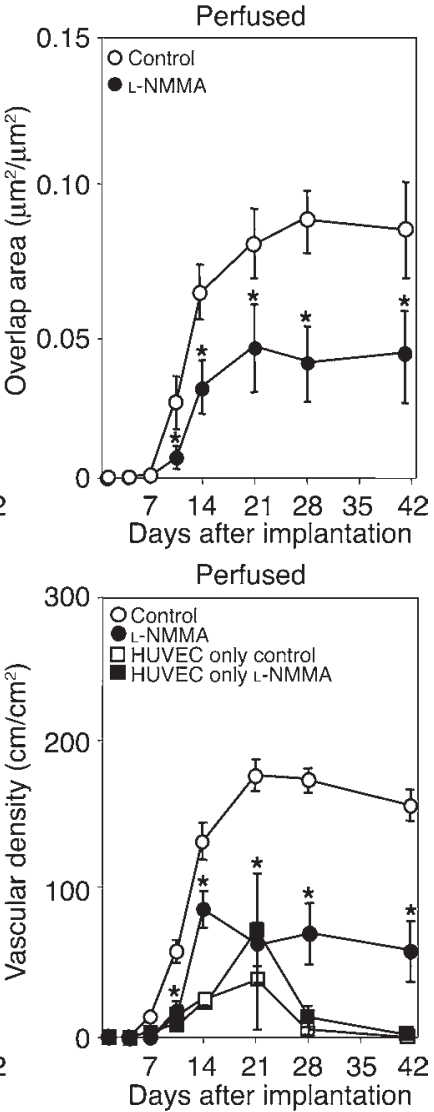

sphingosine-1-phosphate/EDG-1, and angiopoietin/Tie-2, have been shown to induce NO production. NO also has been shown to mediate downstream signaling, angiogenesis, and vascular SMC relaxation induced by these pathways $(7,8,53)$. TGF- $\beta 1$, which has been shown to mediate mural cell differentiation, also stimulates NO production through upregulation of eNOS transcription and stabilization of eNOS mRNA (54). NO may also mediate mural cell recruitment as a second messenger of these signaling pathways.

For the final in vivo analyses we used a newly developed tissue-engineered blood vessel model (29). L-NMMA treatment significantly inhibited the association of 10T1/2 cells with HUVECs and resulted in reduced formation and increased destabilization of the engineered vessels (Figures 9 and 10). Importantly, NO suppression inhibited $10 \mathrm{~T} 1 / 2$ cell recruitment but not proliferation. 10T1/2 cells were present in equal, if not greater, numbers in the L-NMMA-treated group than in the untreated group. However, they did not associate with the HUVEC-derived vessel structure (Figure 9).

The alteration in vessel morphology by NO suppression in the engineered vessels was similar to that in tumor vessels that received the same treatment. Both vessel density and the number of vessel segments were significantly decreased by L-NMMA treatment (Figures 1 and 10). Collectively, the data in this study indicate that endothelial cell-derived NO induces mural cell recruitment to vessels, longitudinal vessel extension, and branching of angiogenic vessels in both B16 melanomas and a tissue-engineered vessel model.

\section{Figure 10}

Endothelial-mural cell interaction and vessel density in the tissue-engineered blood vessels. (A) Temporal changes in the mural cell coverage of endothelial cells. All vessels including both perfused (right) and nonperfused (left) were analyzed. (B) Temporal changes in total (left) and perfused (right) tissue-engineered vessel density. ${ }^{*} P<0.05$, compared with corresponding controls; Mann-Whitney $U$ test. Four locations of each tissue-engineered vessel model were observed. Six animals each for the HUVECs-10T1/2 cells co-implantation models, and 3 animals each for implantation with HUVEC alone were examined.

Tumor vessels are known to have structural and functional abnormalities such as a lack of a hierarchical branching organization, irregular and increased diameter, and increased focal leakiness. These abnormalities play an important role in creating harsh metabolic environments such as hypoxia and acidosis in solid tumors and compromise the delivery of therapeutic agents to tumor cells (55). Clearly, tumor vessels are immature despite the ability of tumors to initiate new blood vessel formation. Proper incorporation of mural cells into the vessel wall is an indispensable step in vessel maturation. Deficiency in mural cell recruitment and defects in these cells' association to tumor vessels are critically involved in the abnormalities of tumor vessels $(45,56)$. The imbalance of angiogenic factors in tumors may cause these abnormalities. Judicious modification of the balance of angiogenic factors may normalize tumor vasculature and potentiate antitumor therapies (55). In this study, the vasculature of B16F10 tumors has more mural cell coverage and branching with relatively smaller vessel diameter, which is indicative of a mature (normal) phenotype, compared with vessels in B16F1 and B16F10 tumors treated with L-NMMA or grown in $\mathrm{eNOS}^{-/-}$mice. These findings suggest that NO produced by eNOS in vascular endothelial cells plays an important role in the regulation of vessel maturation in B16 melanomas and that modulation of NO signaling may be a novel strategy for controlling tumor vessel structure and function.

The inhibition of angiogenesis is a promising new treatment strategy for tumors and other angiogenesis-dependent diseases. However, the efficacy of targeting specific angiogenic factors in tumors may be hampered due to compensation by other angiogenic factors. Since varieties of angiogenic factors and stimuli share $\mathrm{NO}$ as a common signaling pathway, NOS could be a potential target of antiangiogenic therapy that may be applicable to a spectrum of tumors with different expression profiles of angiogenic factors. Caveolin-1 is a negative regulator of eNOS function, and a putative cell-permeable peptide consisting of caveolin scaffolding domain of caveolin-1 (Cavtratin) blocks NO release, vascular permeability, angiogenesis, and tumor growth in implanted human and murine tumors (12).
Table 3

Vascular morphology of tissue-engineered blood vessels

\begin{tabular}{lcccc}
\hline & & & \multicolumn{2}{c}{ All vessels } \\
& \multicolumn{2}{c}{ Perfused vessels } & \multicolumn{2}{c}{ All } \\
& Control & L-NMMA & Control & L-NMMA \\
Vessel diameter $(\mu \mathrm{m})$ & $15.0 \pm 1.1$ & $15.8 \pm 1.3$ & $13.1 \pm 1.1$ & $12.8 \pm 1.3$ \\
No. of vessel segments $\left(\right.$ per mm $\left.{ }^{2}\right)$ & $663 \pm 70$ & $228 \pm 69^{\mathrm{A}}$ & $921 \pm 168$ & $535 \pm 188^{\mathrm{A}}$ \\
Vessel density $\left(\mathrm{cm} / \mathrm{cm}^{2}\right)$ & $177 \pm 9$ & $71 \pm 23^{\mathrm{A}}$ & $237 \pm 10$ & $147 \pm 45^{\mathrm{A}}$ \\
10T1/2 cell coverage $\left(\mu \mathrm{m}^{2} / \mu \mathrm{m}^{2} \times 10^{2}\right)$ & $7 \pm 2$ & $4 \pm 1^{\mathrm{A}}$ & $13 \pm 1$ & $9 \pm 1^{\mathrm{A}}$ \\
\hline
\end{tabular}

Four random locations per animal were analyzed in 6 animals for each group 28 days after implantation. ${ }^{A} P<0.05$ as compared with corresponding controls. 
Many NO effects are mediated via elevation of intracellular cyclic GMP (cGMP) (3, 57). cGMP is generated from GTP either by soluble or membrane-spanning peptide-regulated guanylyl cyclases that abundantly exist in mural cells such as vascular SMCs and pericytes (58). NO activates the former while other stimuli, such as natriuretic peptides, activate the latter. Recently, Yamahara et al. showed that gene transfer of brain natriuretic peptide accelerated neovascularization with proper mural cell coating in ischemic limbs and rescued delayed angiogenesis induced by NO blockade (59). Both NO and C-type natriuretic peptides stimulate primary aortic SMC migration mediated by cGMP (52). Furthermore, genetic alteration of cGMP-dependent protein kinase type I, which is one of the important kinases in the cGMP-signaling pathway, effectively modulated ischemia-induced angiogenesis (59). These findings strongly suggest that the guanylyl cyclase-cGMP pathway is the most plausible downstream pathway of NO-induced angiogenesis and mural cell recruitment. However, in addition, it is increasingly evident that NO mediates cellular signaling via posttranslational modifications of metalloproteins, thiols, and tyrosines (60). It is also possible that other mechanisms such as S-nitrosothiol or nitrotyrosin formation are involved in the angiogenic effects of NO. Dissection of the signaling pathways through which $\mathrm{NO}$ induces angiogenesis and vessel maturation will not only facilitate our understanding of these biological processes but should also suggest novel strategies to control vessel formation and function in tumors and other diseases.

\section{Methods}

Animals and tumors. Two variants of B16 melanoma derived from C57BL/6 mice, B16F1 and B16F10 (61), were maintained in DMEM supplemented with $10 \% \mathrm{FBS}$ at $37^{\circ} \mathrm{C}$ in a humidified $\mathrm{CO}_{2}$ atmosphere. To obtain source tumor tissue, B16 tumor cells in culture $\left(1 \times 10^{6}\right.$ cells $)$ were injected subcutaneously into C57BL/6 mice. When a tumor reached a suitable size $(\sim 8 \mathrm{~mm}$ in diameter), it was excised after euthanasia, and a small piece (about $1 \mathrm{~mm}^{3}$ ) of tumor tissue was implanted into a window model or subcutaneously for an in vivo passage. Serial in vivo passages were limited to fifth generation. Dorsal skin chamber or cranial window preparations in the mice were performed, and a small piece ( $1 \mathrm{~mm}$ in diameter) of B16F10 or B16F1 tumor was implanted into the center of a window in immunodeficient Rag $2^{-/-}$mice (congenic of 129S6/SvEv and C57BL/6) as previously described $(26,27$ ). To determine the role of NOS in host stromal cells, the experiments were performed using B16F10 melanoma grown in C57BL/6 wild-type animals and $\mathrm{NOSS}^{-/-}$or $\mathrm{NOSS}^{-/-}$mice backcrossed for 10 generations to $\mathrm{C} 57 \mathrm{BL} / 6$ background. For the tissue-engineered blood vessel model, SCID mice were used (29). All mice were bred and maintained in either a gnotobiotic animal facility or a barrier animal room in Massachusetts General Hospital under diurnal lighting conditions and allowed free access to food and water. All procedures were carried out following the NIH Public Health Service Policy on Humane Care of Laboratory Animals and approved by the Institutional Animal Care and Use Committee of Massachusetts General Hospital.

Intravital microscopy. Angiogenesis and vessel morphology in B16F10 or B16F1 melanomas grown in the window models were determined by intravital microscopy (27). An upright microscope (Axioplan; Zeiss) equipped with transillumination and fluorescence epi-illumination and an intensified cooled coupled device camera (C2400-88; Hamamatsu) was used. FITClabeled dextran $\left(2 \times 10^{6}\right.$ molecular weight; $10 \mathrm{mg} / \mathrm{ml}, 100 \mu$; Sigma-Aldrich $)$ was injected into the animals' tail veins to contrast enhance functional blood vessels. Five randomly selected locations $\left(500 \times 340 \mu \mathrm{m}^{2}\right.$ of each $)$ of the tumors were visualized, examined by intravital fluorescence microscopy (Zeiss), and recorded through a frame grabber board (Data Translation Inc.) for image digitization on a computer (Apple Computer Inc.). Vascular parameters such as functional vessel density (the total length of perfused microvessels/unit area), vessel diameter, and number of vessel segments (distinct individual vessel segments connected to each other at the branching point) were analyzed by tracing each vessel segment using NIH Image 1.63 freeware on these captured images and by calculating the data using a macro in Microsoft Excel 2003 as described elsewhere (26, 27). The number of vessel segments is an indicator of vessel branching (62). Blood vessels would be divided into many more segments if there were more branches.

NO measurement. NO in B16 melanomas was measured by a polarographic electrochemical method using recessed Nafion-polymer-coated gold microsensors (34) in a procedure described previously (17). Briefly, the windows were opened, and the tumors were superfused with Earle's Balanced Salt Solution (Sigma-Aldrich), equilibrated with $5 \% \mathrm{O}_{2}, 5 \% \mathrm{CO}_{2}$, and balance $\mathrm{N}_{2}$ and maintained at $37^{\circ} \mathrm{C}$. NO microsensors positioned at a $45^{\circ}$ angle with a micromanipulator were advanced into the tumors at a rate of approximately $240 \mu \mathrm{m} / \mathrm{min}$ using a hydraulic microdrive (David Kopf Instruments). Average values deep in the B16F1 and B16F10 tumors (> $250 \mu \mathrm{m})$ grown in the dorsal skin chamber $(59$ profiles in 10 tumors and 61 profiles in 9 tumors, respectively) and those in the cranial window (10 profiles in 2 tumors, 15

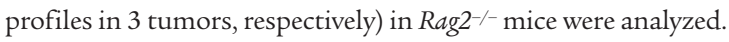

Inbibition of NO production. To lower NO production in the melanomas or the tissue-engineered vessel model, the NOS inhibitor L-NMMA (Alexis Corp.) or nonactive control compound D-NMMA (Alexis Corp.) was given at the rate of $7 \mathrm{mg}$ /day by a constant release micro-osmotic pump (Model 1002, ALZET Osmotic Pumps; Durect Corp.). For the treatment, the microosmotic pumps were implanted in the backs of the animals 1 day before the implantation of tumors or $3 \mathrm{D}$ collagen-fibronectin gels. The pumps were replaced at day 14 in the case of the tissue-engineered vessel model. To determine the role of specific NOSs in host cells, the tumor experiments were performed in wild-type C57BL/6, eNOS $\mathrm{N}^{-/}$, and $i \mathrm{NOS}^{-/-}$mice. All tumors were analyzed 7 days after tumor implantation.

Immunohistochemistry. The following antibodies were used at the dilutions indicated: monoclonal mouse anti-eNOS or anti-nNOS (1:1000, Transduction Laboratory; BD Biosciences - Pharmingen); monoclonal mouse anti-iNOS (1:200, Transduction Laboratory; BD Biosciences - Pharmingen); rat polyclonal MECA-32 (1:200; BD Biosciences - Pharmingen); monoclonal mouse anti- $\alpha$-SMA (1:200, clone 1A4; Sigma-Aldrich); and polyclonal rabbit anti-NG2 (1:1000, Chemicon International). The tumors were excised, fixed in $4 \%$ paraformaldehyde for 4 hours, and embedded in paraffin. Sections (5- $\mu \mathrm{m}$ thick) were immunostained with primary and appropriate secondary antibodies using avidin-biotin complex/diaminobenzidine histochemistry. Slides were analyzed using a BX40 upright microscope (Olympus).

Quantification of endothelial cell number and extent of vessel coverage by mural cells. We performed immunohistochemistry on serial sections stained with hematoxylin as shown in Figure 5A. In the first section, vascular endothelial cells were identified using MECA-32. In the adjacent section, mural cells were identified using anti- $\alpha$-SMA or NG2 antibody staining. Digital images $\left(750 \times 475 \mu \mathrm{m}^{2}\right.$ each) of the immunohistochemistry slides were taken and analyzed using NIH Image 1.63 freeware. We measured the perimeter of each vessel and the length of the vessel segment staining positive for the mural cell marker. The ratio of the mural cell-positive segment to vessel perimeter in each vessel was used to quantify the extent of pericyte coverage. The number of endothelial cell nuclei per vessel cross-section was also quantified. Five locations from each tumor were randomly sampled, and 3 tumors per group were analyzed.

NO bioimaging using NO-sensitive fluorescence probe. NO was visualized using the NO-sensitive fluorescence probe DAF-2 (Daiichi Pure Chemicals Co.) (37) and MPLSM to reveal its spatial and temporal distribution (26). The background fluorescence images were captured before the start of experiments, and then $0.5 \mathrm{mg} /$ body of DAF- 2 was injected intravenously. The 
DAF-2-associated fluorescence increased in a time-dependent manner, and microfluorographs for data analyses were captured 60 minutes after injection. Data calibration of the fluorescence intensities was performed by determining gray levels of known concentrations of DAF-2T (Daiichi Pure Chemicals Co.), a stable compound yielded by the interaction between NO and DAF-2. As shown in Results, the relationship between 8-bit gray level intensities and the concentrations of DAF- $2 \mathrm{~T}$ was established. To reveal the spatial distribution of DAF-2 probe, we also used 4-AF (Daiichi Pure Chemicals Co.), an NO-insensitive fluorochrome. Both DAF-2 and 4-AF are derivatives of fluorescein and have similar molecular weights of 364.3 and 349.3, respectively. Since 4-AF cannot react with $\mathrm{NO}$ via triazole ring formation, the presence of NO does not change its fluorescence intensity, and thus, it has been used as a negative control for DAF-2 (38).

Expression of genes involved in angiogenesis and vessel maturation. Total RNA was extracted from tumors of 6-mm diameter using TRIzol Reagent (Invitrogen Corp.). For cDNA synthesis, TaqMan Reverse Transcription Reagents (Applied Biosystems) were used according to manufacturer's instructions. To screen for relative differential expression of multiple genes, cDNA arrays containing 96 genes involved in angiogenesis and vessel maturation were used according to manufacturer's instructions (GEArray Q Series MM-009, SuperArray Biosciences Corp.). Chemiluminescent spots were quantified by densitometry and normalized with $\beta$-actin (FluoroChem 8800 system). Using real-time PCR, we quantified mRNA levels of various candidate molecules that were identified by cDNA array analysis. LUX Fluorogenic Primers (Invitrogen Corp.) specific for each mRNA species were designed using Invitrogen's D-LUXDesignerSoftware (primersequences are listed in Supplemental TableS4). Quantitative RT-PCRs were performed on the Applied Biosystems 7300 RealTime PCR System (Applied Biosystems). All experiments were performed in duplicate, and a standard curve for the specific cDNA of interest was run with every PCR reaction; amounts of cDNA are expressed relative to this standard curve. Final quantification of each cDNA sample was relative to mouse $\beta$-actin following the manufacturer's instructions (Applied Biosystems).

Cell culture. HUVECs were provided by M. Gimbrone (Brigham and Women's Hospital, Harvard Medical School, Boston, Massachusetts, USA) and maintained in endothelial growth media (EGM) (Cambrex) with 12\% FBS and bovine brain extract (Cambrex), and used through passage 5. 10T1/2 cells (no. CCL 226; ATCC) were grown in basal medium Eagle (BME) with $10 \% \mathrm{FBS}$ and $2 \mathrm{mM}$ glutamine. All cells were maintained at $37^{\circ} \mathrm{C}$ in a humidified $5 \% \mathrm{CO}_{2}$ incubator. HUVECs and $10 \mathrm{~T} 1 / 2$ cells were transfected with eGFP or DsRed gene using retroviral vectors as described (29).

Transwell migration assay. Cell migration was assessed using Falcon HTS FluoroBlok 24-well inserts (BD Biosciences) with $1-\mu \mathrm{m}$ pores. eGFP-10T1/2 cells $\left(2 \times 10^{4}\right)$ suspended in 250- $\mu$ l BME (Invitrogen Corp.) with $0.5 \%$ FBS were placed inside each insert while $5 \times 10^{4}$ per well HUVECs suspended in $800 \mu \mathrm{l}$ EGM (Cambrex) were plated on a 24-well plate. Eight hours later, all cell culture media were changed to BME with $0.5 \% \mathrm{FBS}$, and then the inserts were placed in the respective wells. To examine the effect of NO inhibition on cell migration, 10 or $100 \mu \mathrm{M}$ of L-NMMA was added to the medium. After 8 hours, a $15-\mathrm{mm}^{2}$ area outside the bottom of each insert was imaged in fluorescence using a mosaic image acquisition pattern via a custom-made algorithm as described before (63) using an inverted fluorescence microscope (Olympus IX70) equipped with a motorized stage and motorized filter wheel (Improvision Inc.). Transmigrated cells were quantified using the thresholding and measure particle functions of NIH Image 1.63 freeware.

Under-agarose migration assay. This assay allows for the interaction of the 2 cell types via their soluble products, and the directional migration of both cell types under the agarose gel can be quantified with image analysis (28). Two 4-mm-diameter wells $2.5 \mathrm{~mm}$ apart were created in 1\% agarose in EGM using cloning cylinders during gelation. HUVECs and eGFP-10T1/2 cells were plated individually in wells at $5 \times 10^{4}$ cells/well in $100-\mu l$ low-serum $(2 \%)$
EGM media to reduce cell proliferation. To inhibit NO, $100 \mu \mathrm{M}$ of L-NMMA was incorporated in both the agarose gel and the culture media inside the wells. Cells were cultured for 4 days at $37^{\circ} \mathrm{C}$ before quantification of cell migration. Images of the area around and between the wells were acquired in a mosaic acquisition pattern both in brightfield and fluorescence as described above. During the 4-day coculture, cells migrated but did not make direct contact. The migration distance from the edge of the well and the number of migrated cells were measured using NIH Image 1.63 freeware.

To enable assessment of proliferation activity, cells were subsequently fixed in 4\% paraformaldehyde and immunostained with mouse monoclonal antibody to PCNA (PC10, DakoCytomation Inc.) at a dilution of 1:50 followed by application of Texas Red Donkey-labeled anti-mouse IgG2a secondary antibody (Jackson ImmunoResearch Laboratories Inc.) and DAPI (Invitrogen Corp.). Images of these cells were acquired using an inverted fluorescence microscope (Olympus) for analysis. All stained nuclei were scored positive for PCNA. The PCNA-labeling index (number of positive nuclei/total number of nuclei) was determined by observation of more than 1000 nuclei for each experimental sample and was used for evaluating the proliferating activity of the cells.

Tissue-engineered vessel model. The tissue-engineered blood vessel model was prepared as described (29). Briefly, $8 \times 10^{5}$ eGFP-HUVECs and $2 \times 10^{5}$ of DsRed-10T1/2 cells or $1 \times 10^{6}$ eGFP-HUVECs alone were suspended in $1 \mathrm{ml}$ of type 1 collagen-fibronectin solution and solidified in 12 -well plates (BD Biosciences). After 1 day of culture, circular disk-shaped pieces of the construct (4-mm diameter) were created by a skin puncher and implanted into the cranial windows in SCID mice. To lower NO production in the model, $100 \mu \mathrm{M}$ L-NMMA was incorporated in the gel, and the animals were treated as described above. MPLSM was used to visualize and document the interaction of eGFP-HUVECs and DsRed-10T1/2 cells as well as the formation and alteration of engineered vessels $(26,29)$. Functional vessels were contrast-enhanced by an injection of $1 \%$ tetramethylrhodaminelabeled dextran $\left(2 \times 10^{6}\right.$ molecular weight). Vascular parameters such as functional vessel density, total length of perfused microvessels per unit area, vessel diameter, and number of individual segments were analyzed using NIH image 1.63 freeware $(26,29)$. The extent of $10 \mathrm{~T} 1 / 2$ cell coverage of HUVECs was determined by use of an NIH image 1.63 freeware macro as shown in Figure 9B. Briefly, the macro identified eGFP-HUVECs and DsRed-10T1/2 cells and binarized them, then the diameters of the binarized HUVECs areas were dilated by $4 \mu \mathrm{m}$ from the original edges. Overlap of dilated endothelial cell area with 10T1/2 cell area was considered 10T1/2 cell coverage over vessels. These parameters were determined in 4 photographic areas $\left(275 \times 367 \mu \mathrm{m}^{2}\right.$ each $)$ of each engineered vessel model.

Statistics. The data were analyzed by analysis of variance and Fisher's post hoc test using StatView (SAS Institute Inc.) unless otherwise specified. Values are expressed as mean \pm SEM unless otherwise specified. Statistical significance was set at $P<0.05$.

\section{Acknowledgments}

We thank Leo Gerweck, Jeroen Hagendoorn, and Wilson Mok for their insightful suggestions; Emmanuelle di Tomaso for her assistance in immunohistochemistry; Naoto Koike and Patrick Au for their assistance in the tissue-engineered blood vessel model; Peigen Huang for his direction of the gnotobiotic animal facility; Julia Kahn, Michele Riley, Sylvie Roberge, Carolyn Smith, Melanie Fortier, Russell Delgiacco, and Christopher M. VanWart for their excellent technical assistance; and Tara Belezos for preparation of the manuscript. This work was supported in part by NIH grants R24-CA85140 (to R.K. Jain and D. Fukumura), RO1-CA96915 (to D. Fukumura), and HL-068164 (to D.G. Buerk), and NSF grant BES-0301446 (to D.G. Buerk). 
Received for publication November 29, 2004, and accepted in revised form May 3, 2005.
COX-7, Boston, Massachusetts 02114, USA. Phone: (617) 726-8143; Fax: (617) 724-5841; E-mail: dai@steele.mgh.harvard.edu.

Address correspondence to: Dai Fukumura, Department of Radia- Yotaro Izumi and Takeshi Gohongi contributed equally to this tion Oncology, Massachusetts General Hospital, 100 Blossom Street, work.

1. Moncada, S. 1997. Nitric oxide in the vasculature: physiology and pathophysiology. Ann. N. Y. Acad. Sci. 811:60-67.

2. Nathan, C., and Xie, Q.-W. 1994. Nitric oxide synthases: roles, tolls, and controls. Cell. 78:915-918.

3. Ignarro, L.J., Cirino, G., Casini, A., and Napoli, C. 1999. Nitric oxide as a signaling molecule in the vascular system: an overview. J. Cardiovasc. Pharmacol. 34:879-886.

4. Fukumura, D., and Jain, R.K. 1998. Role of nitric oxide in angiogenesis and microcirculation in tumors. Cancer Metastasis Rev. 17:77-89.

5. Duda, D.G., Fukumura, D., and Jain, R.K. 2004. Role of eNOS in neovascularization: NO for endothelial progenitor cells [review]. Trends Mol. Med. 10:143-145.

6. van der Zee, R., et al. 1997. Vascular endothelial growth factor/vascular permeability factor augments nitric oxide release from quiescent rabbit and human vascular endothelium. Circulation. 95:1030-1037.

7. Babaei, S., et al. 2003. Angiogenic actions of angiopoietin-1 require endothelium-derived nitric oxide. Am.J. Pathol. 162:1927-1936.

8. Igarashi, J., Bernier, S.G., and Michel, T. 2001. Sphingosine 1-phosphate and activation of endothelial nitric-oxide synthase. differential regulation of Akt and MAP kinase pathways by EDG and bradykinin receptors in vascular endothelial cells. J. Biol. Chem. 276:12420-12426.

9. Dimmeler, S., et al. 1999. Activation of nitric oxide synthase in endothelial cells by Akt-dependent phosphorylation. Nature. 399:601-605.

10. Dulak, J., et al. 2000. Nitric oxide induces the synthesis of vascular endothelial growth factor by rat vascular smooth muscle cells. Arterioscler. Thromb. Vasc. Biol. 20:659-666.

11. Ziche, M., et al. 1997. Nitric oxide promotes proliferation and plasminogen activator production by cornoary venular endothliuim through endogenous bFGF. Circ. Res. 80:845-852.

12. Gratton, J.P., et al. 2003. Selective inhibition of tumor microvascular permeability by cavtratin blocks tumor progression in mice. Cancer Cell. 4:31-39.

13. Gallo, O., et al. 1998. Role of nitric oxide in angiogenesis and tumor progression in head and neck cancer. J. Natl. Cancer Inst. 90:584-596.

14. Lorraine, J.C., and Lala, P.K. 1999. Nitric oxide synthase inhibition by NG-nitro-L-arginine methyl ester inhibits tumor-induced angiogenesis in mammary tumors. Am. J. Pathol. 155:1381-1390.

15. Lee, P.C., et al. 1999. Impaired wound healing and angiogenesis in eNOS-deficient mice. Am. J. Physiol. 277:H1600-H1608.

16. Murohara, T., et al. 1998. Nitric oxide synthase modulates angiogenesis in response to tissue ischemia. J. Clin. Invest. 101:2567-2578.

17. Fukumura, D., et al. 2001. Predominant role of endothelial nitric oxide synthase in vascular endothelial growth factor-induced angiogenesis and vascular permeability. Proc. Natl. Acad. Sci. U. S. A. 98:2604-2609.

18. Ando, A., et al. 2002. Nitric oxide is proangiogenic in the retina and choroid. J. Cell. Physiol. 191:116-124.

19. Wang, B., et al. 2001. Genetic disruption of host nitric oxide synthase II gene impairs melanoma-induced angiogenesis and suppresses pleural effusion. Int. J. Cancer. 91:607-611.

20. Konopka, T.E., et al. 2001. Nitric oxide synthase II gene disruption: implication for tumor growth and vascular endothelial growth factor production. Cancer Res. 61:3182-3187.

21. Hirschi, K.K., and D'Amore, P.A. 1996. Pericytes in the microvasculature. Cardiovasc. Res. 32:687-698.
22. Jain, R.K. 2003. Molecular regulation of vessel maturation. Nat. Med. 9:685-693.

23. Gerhardt, H., and Betsholtz, C. 2003. Endothelialpericyte interactions in angiogenesis. Cell Tissue Res. 314:15-23.

24. Perlis, C., and Herlyn, M. 2004. Recent advances in melanoma biology. Oncologist. 9:182-187.

25. Hsu, M.-Y., Meier, F., and Herlyn, M. 2002. Melanoma development and progression: a conspiracy between tumor and host. Differentiation. 70:522-536.

26. Jain, R.K., Brown, E.B., Munn, L.L., and Fukumura, D. 2004. Intravital microscopy of normal and diseased tissues in mice. In Live cell imaging: a laboratory manual. R. Goldman and D. Spector, editors. Cold Spring Harbor Laboratory Press. Cold Spring Harbor, New York, USA. 435-466.

27. Jain, R.K., Munn, L.L., and Fukumura, D. 2002. Dissecting tumour pathophysiology using intravital microscopy. Nat. Rev. Cancer. 2:266-276.

28. Hirschi, K.K., Rohovsky, S.A., and D'Amore, P.A. 1998. PDGF, TGF-beta, and heterotypic cell-cell interactions mediate endothelial cell-induced recruitment of $10 \mathrm{~T} 1 / 2$ cells and their differentiation to a smooth muscle fate. J. Cell Biol. 141:805-814.

29. Koike, N., et al. 2004. Tissue engineering: creation of long-lasting blood vessels. Nature. 428:138-139.

30. Folkman, J., and Kalluri, R. 2004. Cancer without disease. Nature. 427:787.

31. Carmeliet, P., and Jain, R.K. 2000. Angiogenesis in cancer and other diseases: from genes to function to therapy. Nature. 407:249-257.

32. Fidler, I.J., and Ellis, L.M. 1994. The implications of angiogenesis for the biology and therapy of cancer metastasis. Cell. 79:185-188.

33. Ziche, M., et al. 1994. Nitric oxide mediates angiogenesis in vivo and endothelial cell growth and migration in vitro promoted by substance P. J. Clin. Invest. 94:2036-2044.

34. Buerk, D.G., Riva, C.E., and Cranstoun, S.D. 1996. Nitric oxide has a vasodilatory role in rat optic nerve head during flicker stimuli. Microvasc. Res. 52:13-26.

35. Fulton, D., Gratton, J.P., and Sessa, W.C. 2001. Posttranslational control of endothelial nitric oxide synthase: Why isn't calcium/calmodulin enough? J. Pharmacol. Exp. Ther. 299:818-824.

36. Aktan, F. 2004. iNOS-mediated nitric oxide production and its regulation. Life Sci. 75:639-653.

37. Kojima, H., et al. 1998. Development of a fluorescent indicator for nitric oxide based on the fluorescein chromophore. Chem. Pharm. Bull. 46:373-375.

38. Kojima, H., et al. 1998. Direct evidence of NO production in rat hippocampus and cortex using a new fluorescent indicator: DAF-2 DA. Neuroreport. 9:3345-3348.

39. Blute, T.A., Lee, M.R., and Eldred, W.D. 2000. Direct imaging of NMDA-stimulated nitric oxide production in the retina. Vis. Neurosci. 17:557-566.

40. Darland, D.C., and D'Amore, P.A. 2001. Cell-cell interactions in vascular development. Curr. Top. Dev. Biol. 52:107-149.

41. Kashiwagi, S., Kaijimura, M., Yoshimura, Y., and Suematsu, M. 2002. Nonendothelial source of nitric oxide in arterioles but not in venules. Circ. Res. 91:e55-e64.

42. Jain, R.K. 1988. Determinants of tumor blood flow: a review. Cancer Res. 48:2641-2658.

43. Ambs, S., et al. 1998. p53 and vascular endothelial growth factor regulate tumor growth of NOS2-expressing human carcinoma cells. Nat. Med. 4:1371-1376.

44. Papapetropoulos, A., Garcia-Cardena, G., Madri, J.A., and Sessa, W.C. 1997. Nitric oxide production contributes to the angiogenic properties of vascular endothelial growth factor in human endothelial cells. J. Clin. Invest. 100:3131-3139.

45. Morikawa, S., et al. 2002. Abnormalities in pericytes on blood vessels and endothelial sprouts in tumors. Am. J. Pathol. 160:985-1000.

46. Hirschi, K.K., Rohovsky, S.A., Beck, L.H., Smith, S.R., and D'Amore, P.A. 1999. Endothelial cells modulate the proliferation of mural cell precursors via plateletderived growth factor-BB and heterotypic cell contact. Circ. Res. 84:298-305.

47. Schlingemann, R.O., et al. 1991. Differential expression of markers for endothelial cells, pericytes, and basal lamina in the microvasculature of tumors and granulation tissue. Am. J. Pathol. 138:1335-1347.

48. Liu, Y., et al. 2000. Edg-1, the G protein-coupled receptor for sphingosine-1-phosphate, is essential for vascular maturation. J. Clin. Invest. 106:951-961.

49. Suri, C., et al. 1996. Requisite role of angiopoietin-1, a ligand for the TIE2 receptor, during embryonic angiogenesis. Cell. 87:1171-1180.

50. Lindahl, P., Johansson, B.R., Leveen, P., and Betsholtz, C. 1997. Pericyte loss and microaneurysm formation in PDGF-B-deficient mice. Science. 277:242-245.

51. Sreejayan, N., Lin, Y., and Hassid, A. 2002. NO attenuates insulin signaling and motility in aortic smooth muscle cells via protein tyrosine phosphatase 1Bmediated mechanism. Arterioscler. Thromb. Vasc. Biol. 22:1086-1092.

52. Brown, C., Pan, X., and Hassid, A. 1999. Nitric oxide and C-type atrial natriuretic peptide stimulate primary aortic smooth muscle cell migration via a cGMPdependent mechanism: relationship to microfilament dissociation and altered cell morphology. Circ. Res. 84:655-667.

53. Cunningham, L.D., Brecher, P., and Cohen, R.A. 1992. Platelet-derived growth factor receptors on macrovascular endothelial cells mediate relaxation via nitric oxide in rat aorta. J. Clin. Invest. 89:878-882.

54. Inoue, N., et al. 1995. Molecular regulation of the bovine endothelial cell nitric oxide synthase by transforming growth factor-beta 1. Arterioscler. Thromb. Vasc. Biol. 15:1255-1261.

55. Jain, R.K. 2005. Normalization of tumor vasculature: an emerging concept in antiangiogenic therapy [review]. Science. 307:58-62.

56. Abramsson, A., et al. 2002. Analysis of mural cell recruitment to tumor vessels. Circulation. 105:112-117.

57. Moncada, S. 1992. The L-arginine: nitric oxide pathway. Acta Physiol. Scand. 145:201-227.

58. Joyce, N.C., DeCamilli, P., and Boyles, J. 1984. Pericytes, like vascular smooth muscle cells, are immunocytochemically positive for cyclic GMP-dependent protein kinase. Microvasc. Res. 28:206-219.

59. Yamahara, K., et al. 2003. Significance and therapeutic potential of the natriuretic peptides/cGMP/cGMPdependent protein kinase pathway in vascular regeneration. Proc. Natl. Acad. Sci. U. S. A. 100:3404-3409.

60. Gow, A.J., Farkouh, C.R., Munson, D.A., Posencheg, M.A., and Ischiropoulos, H. 2004. Biological significance of nitric oxide-mediated protein modifications. Am. J. Physiol. Lung Cell. Mol. Physiol. 287:L262-L268.

61. Fidler, I. 1973. Selection of successive tumour lines for metastasis. Nat. New Biol. 242:148-149.

62. Fukumura, D., et al. 2003. Paracrine regulation of angiogenesis and adipocyte differentiation during in vivo adipogenesis. Circ. Res. 93:e88-e97.

63. Demou, Z.N., and McIntire, L.V. 2002. Fully automated three-dimensional tracking of cancer cells in collagen gels: determination of motility phenotypes at the cellular level. Cancer Res. 62:5301-5307. 